\title{
Invisible neutrino decay: first vs second oscillation maximum
}

\author{
Kaustav Chakraborty, ${ }^{a}$ Debajyoti Dutta, ${ }^{b}$ Srubabati Goswami ${ }^{a}$ and \\ Dipyaman Pramanik ${ }^{a, c}$ \\ ${ }^{a}$ Theoretical Physics Division, Physical Research Laboratory, \\ Ahmedabad, 380009, India \\ ${ }^{b}$ Assam Don Bosco University, \\ Tapesia Campus, Sonapur, Assam, 782402, India \\ ${ }^{c}$ Instituto de Física Gleb Wataghin - UNICAMP, \\ 13083-859, Campinas, São Paulo, Brazil \\ E-mail: kaustav@prl.res.in, debajyoti.dutta@dbuniversity.ac.in, \\ sruba@prl.res.in, dipyaman@unicamp.br
}

ABSTRACT: We study the physics potential of the long-baseline experiments T2HK, T2HKK and ESS $\nu \mathrm{SB}$ in the context of invisible neutrino decay. We consider normal mass ordering and assume the state $\nu_{3}$ as unstable, decaying into sterile states during the flight and obtain constraints on the neutrino decay lifetime $\left(\tau_{3}\right)$. We find that T2HK, T2HKK and $\operatorname{ESS} \nu \mathrm{SB}$ are sensitive to the decay-rate of $\nu_{3}$ for $\tau_{3} / m_{3} \leq 2.72 \times 10^{-11} \mathrm{~s} / \mathrm{eV}$, $\tau_{3} / m_{3} \leq 4.36 \times 10^{-11} \mathrm{~s} / \mathrm{eV}$ and $\tau_{3} / m_{3} \leq 2.43 \times 10^{-11} \mathrm{~s} / \mathrm{eV}$ respectively at $3 \sigma$ C.L. We compare and contrast the sensitivities of the three experiments and specially investigate the role played by the mixing angle $\theta_{23}$. It is seen that for experiments with flux peak near the second oscillation maxima, the poorer sensitivity to $\theta_{23}$ results in weaker constraints on the decay lifetime. Although, T2HKK has one detector close to the second oscillation maxima, having another detector at the first oscillation maxima results in superior sensitivity to decay. In addition, we find a synergy between the two baselines of the T2HKK experiment which helps in giving a better sensitivity to decay for $\theta_{23}$ in the higher octant. We discuss the octant sensitivity in presence of decay and show that there is an enhancement in sensitivity which occurs due to the contribution from the survival probability $P_{\mu \mu}$ is more pronounced for the experiments at the second oscillation maxima. We also obtain the combined sensitivity of T2HK+ESS $\nu \mathrm{SB}$ and T2HKK $+\mathrm{ESS} \nu \mathrm{SB}$ as $\tau_{3} / m_{3} \leq 4.36 \times 10^{-11} \mathrm{~s} / \mathrm{eV}$ and $\tau_{3} / m_{3} \leq 5.53 \times 10^{-11} \mathrm{~s} / \mathrm{eV}$ respectively at $3 \sigma$ C.L.

Keywords: Beyond Standard Model, Neutrino Physics, CP violation

ArXiv EPrint: 2012.04958 


\section{Contents}

1 Introduction 1

2 Neutrino oscillation in presence of invisible decay of neutrino 4

3 Experimental and simulation details 4

3.1 Experimental details 5

3.1.1 T2HK 5

3.1.2 T2HKK 5

3.1.3 $\mathrm{ESS} \nu \mathrm{SB} \quad 5$

3.2 Simulation details 6

4 Results $\quad 7$

4.1 Probability at T2HK/T2HKK and ESS $\nu$ SB baselines 9

$\begin{array}{lll}4.2 & \text { Sensitivity to the decay } & 10\end{array}$

4.3 Synergy between $295 \mathrm{~km}$ and $1100 \mathrm{~km}$ baselines $\quad 12$

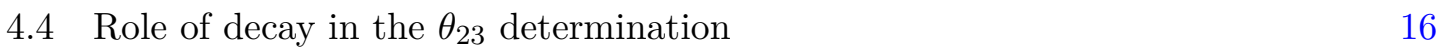

$\begin{array}{lll}4.5 & \text { Combined analysis } & 17\end{array}$

5 Summary \& conclusions $\quad 20$

\section{Introduction}

Neutrinos are one of the most fascinating particles in nature. Observation of oscillation of neutrinos signifies evidence of neutrino mass. The very existence of neutrino mass is the reason of neutrinos being so special since in the Standard Model (SM) of particle physics there is no explanation of neutrino mass. The canonical formalism of neutrino oscillation requires three mixing angles $\theta_{12}, \theta_{13}$ and $\theta_{23}$, two mass-squared differences $\Delta m_{21}^{2}$ and $\Delta m_{31}^{2}$ and one CP phase $\delta_{C P}$. The parameters $\theta_{12}, \theta_{13}, \Delta m_{21}^{2}$ and the absolute value of $\Delta m_{31}^{2}$ have been measured very precisely by oscillation experiments. However, the octant of $\theta_{23}$, the sign of $\Delta m_{31}^{2}$ (mass-ordering) and $\delta_{C P}$ are not yet determined with certainty. Global analysis results [1-3] indicate towards normal hierarchy of $\Delta m_{31}^{2}$ and $\theta_{23}$ in the higher octant. The global analysis also disfavours $\delta_{C P}=\frac{\pi}{2}$ with more than $3 \sigma$ CL.

Many experiments are planned for the near future with the goal of determining these with enhanced precision. Some examples of future experiments are DUNE [4-8], T2HK/ T2HKK [9, 10], ESS $\nu$ SB [11, 12], JUNO [13], INO [14], PINGU [15], KM3Net-ORCA [16] etc. It is a well known fact that if there is a new physics beyond the SM, then that can result in modification of the oscillation probabilities in these experiments. So, these experiments can be sensitive to the new physics. Also, the new physics can affect the sensitivity of these 
experiments to the standard parameters. Invisible neutrino decay during the flight is one such new physics idea.

The idea of neutrino decay was first proposed to explain the solar neutrino problem in the very early days [17]. Later, neutrino oscillation with decay solutions were studied as an explanation of the depletion of solar neutrinos [18-25]. These assumed $\nu_{2}$ to be the unstable state and were able to put bound on the lifetime of $\nu_{2}$. The bound from the solar neutrino data is $\tau_{2} / m_{2}>8.5 \times 10^{-7} \mathrm{~s} / \mathrm{eV}$ [24]. A recent study analysed low energy solar neutrino data and put bounds on both $\tau_{2}$ and $\tau_{1}$ [26]. Supernova neutrinos also give bounds on $\tau_{1}$ and $\tau_{2}$. SN1987A data gives the bound of $\tau / m>10^{5} \mathrm{~s} / \mathrm{eV}$ [27]. $\tau_{1}$ and $\tau_{2}$ can also be constrained from high resolution multi-ton Xenon detector. Recently, in ref. [28], the authors have shown that such a detector can give very strong bounds $\tau_{1} / m_{1} \gtrsim 3 \times 10^{-2}$ $\mathrm{s} / \mathrm{eV}$ and $\tau_{2} / m_{2} \gtrsim 8 \times 10^{-3} \mathrm{~s} / \mathrm{eV}$ at $2 \sigma$ level using solar neutrinos.

Atmospheric and long-baseline experiments give bounds on the $\nu_{3}$ lifetime. A neutrino decay solution (without any oscillation) was proposed in ref. [29] to explain the atmospheric neutrino problem but this solution fitted the data poorly. Refs. [30, 31] considered neutrino decay and neutrino mixing together. This was successful to reproduce the $L / E$ distribution of the Super-Kamiokande (SK) data. However, when zenith angle dependence was used instead of L/E distribution, this model was found to give poorer fit to the SK data [32]. Refs. [30, 32] assumed $\Delta m^{2}>0.1 \mathrm{eV}^{2}$ to comply with the K-decay bounds [30]. Therefore $\Delta m^{2}$ dependent terms were averaged out. These constraints can be relaxed if the unstable state decays to some invisible state with which it has no mixing. In this context, two scenarios have been studied in the literature. The first kept $\Delta m^{2}$ unconstrained and it explicitly appeared in the probabilities [33]. This fits the SK data with a best-fit value of non-zero decay parameter and $\Delta m^{2} \sim 0.003 \mathrm{eV}^{2}$. Ref. [34] considered $\Delta m^{2} \ll 10^{-4} \mathrm{eV}^{2}$. Here, the probability does not contain $\Delta m^{2}$ explicitly. This was able to fit the SK data. However, independent analysis by SK collaboration showed that this scenario gives a poorer fit to the data than only oscillation [35]. Global analysis of atmospheric and long-baseline experiments were performed in ref. [36]. Only oscillation gave best-fit to the SK data and the fit for the oscillation plus decay was not bad. But addition of LBL data from MINOS reduced the fit quality. This analysis put bound on $\tau_{3} / m_{3} \geq 2.9 \times 10^{-10} \mathrm{~s} / \mathrm{eV}$ at the 90 $\%$ C.L. Ref. [37] studied the oscillation plus decay scenario with unconstrained $\Delta m^{2}$ for MINOS and T2K data and it found $\tau_{3} / m_{3}>2.8 \times 10^{-12} \mathrm{~s} / \mathrm{eV}$ at $90 \%$ C.L. Most of these analyses are done using two generation approximation without matter effect. Authors of ref. [38] performed a complete three generation study of the oscillation plus decay scenario assuming matter effect for the NOvA and T2K preliminary data. This study put a bound on the lifetime on the $\tau_{3} / m_{3}$ as $\tau_{3} / m_{3} \geq 1.50 \times 10^{-12} \mathrm{~s} / \mathrm{eV}$ at $3 \sigma$. Recently, ref. [39] addressed the IceCUBE track and cascade tension using invisible neutrino decay. They showed that an unstable neutrino with $\tau / m=100 \mathrm{~s} / \mathrm{eV}$ solves the track vs cascade tension.

There have been many studies in literature which discuss the potential of future experiments to the invisible decay. Medium baseline reactor neutrino experiment like JUNO can give a bound on $\tau_{3} / m_{3}>7.5 \times 10^{-11} \mathrm{~s} / \mathrm{eV}$ (95 \% C.L.) [40]. Decay of ultrahigh energy astrophysical neutrinos can give constraints on decay [41-44]. Recently, ref [45] showed that IceCUBE can probe $\tau / m$ upto $10 \mathrm{~s} / \mathrm{eV}$ for both mass-orderings for $100 \mathrm{TeV}$ neutrinos 
coming from a source at a distance of $1 \mathrm{Gpc}$. The ref. [46] studied the invisible neutrino decay in the context of DUNE. It showed that using charged-current electron type and muon type events, DUNE after $5+5$ years of running can put a bound $\tau_{3} / m_{3}>4.50 \times 10^{-11}$ $\mathrm{s} / \mathrm{eV}$ at $90 \%$ confidence level. More recently ref. [47] performed a multi-channel analysis using charged-current, neutral current and tau-channel events and showed that DUNE's sensitivity would be $\tau_{3} / m_{3}>5.2 \times 10^{-11} \mathrm{~s} / \mathrm{eV}$ at $90 \%$ confidence level. For the potential of MOMENT experiment to probe invisible neutrino decay see ref [48]. There are also studies on the expected sensitivities from the future atmospheric experiments. See refs. [49, 50] for INO and ref. [51] for KM3Net-ORCA. Invisible neutrino decay can happen for both Dirac and Majorana neutrinos. If neutrinos are Dirac, there can be a coupling between the neutrinos and a light scalar boson $[18,52]$. This gives the decay channel $\nu_{j} \rightarrow \bar{\nu}_{i R}+\chi$, where $\bar{\nu}_{i R}$ is a right-handed singlet and $\chi$ is an iso-singlet scalar. If neutrinos are Majorana particles, neutrino can couple with a Majoron and a sterile neutrino via a pseudo-scalar coupling $[53,54]$. This gives $\nu_{j} \rightarrow \nu_{s}+J$. LEP data on the Z-decay to invisible particles constraints the Majoron to be dominantly singlet [55]. Neutrinos can also decay to another active state [56-58]. This is called the visible neutrino decay scenario. This type of decay can happen in the following way. $\nu_{j} \rightarrow \bar{\nu}_{i}+J$ or $\nu_{j} \rightarrow \nu_{i}+J$. If neutrinos are Majorana, the decay product can be observed in the detector. Refs. [59-61] discusses the visible decay in the context of long-baseline experiments. In ref. [62], the authors discuss the visible neutrino decay for reactor experiments KAMLAND and JUNO. For visible decay of the astrophysical neutrinos at IceCUBE, see ref. [63].

Invisible neutrino decay can be constrained using Cosmological observations. In ref. [64], the authors put a bound on neutrino lifetime $\tau_{\nu}>(1.3-0.3) \times 10^{9} \mathrm{~s}\left(m_{\nu} / 0.05 \mathrm{eV}\right)^{3}$ at $95 \%$ C.L. using Planck2018 data.

In this paper we study the constraints on invisible neutrino decay which can come from future planned/proposed long baseline experiments - T2HK/T2HKK $[9,10]$ and $\mathrm{ESS} \nu \mathrm{SB}[12]$. We perform a full three flavour study using matter effect and obtain the sensitivity to $\tau_{3} / m_{3}$ for these experiments. The salient feature of the T2HKK and ESS $\nu$ SB experiment is that they are both designed to have energy peak near the second-oscillation maximum. Since the second oscillation maximum occurs at a lower energy for a particular baseline the effect of decay is expected to be more. We examine this aspect and delve into the detail of whether the experiments at the second oscillation maximum stand to gain in sensitivity in presence of decay. We also check if the determination of $\theta_{23}$ can get affected if we assume decay in the data, while the fit does not assume any decay to be present. In particular, we investigate how the experiments at first and second oscillation maximum fare in this respect and what are the important factors on which the measurement of $\theta_{23}$ can depend in presence of decay. We also explore how the octant sensitivity of these experiments change in presence of neutrino decay.

The paper is organized in the following way. The next section discusses the neutrino oscillation in presence of invisible neutrino decay. In section 3 we give our experimental and numerical details, in section 4 we present our results and in section 5 , we finally draw our conclusions. 


\section{Neutrino oscillation in presence of invisible decay of neutrino}

In this section we discuss the propagation of neutrinos in presence of invisible neutrino decay. We assume that the $\nu_{3}$ is unstable and it decays into a sterile neutrino and a singlet scalar $\left(\nu_{3} \rightarrow \bar{\nu}_{4}+J\right)$ with lifetime $\tau_{3}$. In this case we can extend the mass and flavour bases by $\left(\begin{array}{ll}\nu_{i} & \nu_{4}\end{array}\right)^{T}$ and $\left(\begin{array}{ll}\nu_{\alpha} & \nu_{s}\end{array}\right)^{T}$, where $i=1,2,3$ and $\alpha=\nu_{e}, \nu_{\mu}, \nu_{\tau}$. They are related by the following unitary relation.

$$
\left(\begin{array}{l}
\nu_{\alpha} \\
\nu_{s}
\end{array}\right)=\left(\begin{array}{ll}
U & 0 \\
0 & 1
\end{array}\right)\left(\begin{array}{l}
\nu_{i} \\
\nu_{4}
\end{array}\right) .
$$

$U$ is the PMNS matrix describing the standard 3 neutrino oscillation. We assume normal hierarchy and $m_{4}$ to be the least massive state. We assume that the decay eigenstates and the mass-eigenstates are same. Under these assumptions, we can write the neutrino evolution in presence of matter in the following way.

$$
i \frac{d}{d x} \nu_{f}=\frac{1}{2 E}\left[U \tilde{H} U^{\dagger}+A\right] \nu_{f}
$$

Where,

$$
\tilde{H}=\left(\begin{array}{ccc}
0 & 0 & 0 \\
0 & \Delta m_{21}^{2} & 0 \\
0 & 0 & \Delta m_{31}^{2}-i \frac{m_{3}}{\tau_{3}}
\end{array}\right),
$$

and

$$
A=2 \sqrt{2} G_{F} n_{e} E .
$$

Here, A is the matter potential, $G_{F}$ is the Fermi constant, $E$ is the energy and $n_{e}$ density of electrons in the earth. We define $\alpha_{3}=m_{3} / \tau_{3}$ as the decay rate of the $\nu_{3}$ state. Since we are considering the decay of $\nu_{3}$ only, from here onward, we use $\alpha$ instead of $\alpha_{3}$ for the $\nu_{3}$ state. The probability of getting a neutrino in the flavour state $\nu_{b}$ for the initial flavour state of $\nu_{a}$ is given by

$$
P_{a b}=\left|\left\langle\nu_{b} \mid \nu_{a}\right\rangle\right|^{2}
$$

Here, a,b denote the flavour states e, $\mu, \tau$.

The effect of decay comes as the $\exp (-\alpha L / E)$ factor in the probability. So an experiment is sensitive to the values of $\alpha$ where, $\alpha \sim E / L$. It is clear that, smaller values of $\alpha$ can be probed and hence a higher sensitivity can be achieved for longer baselines and for a particular baseline the sensitivity is more for a lower energy. Thus, sensitivity is expected to be more at the second oscillation maxima as compared to the first oscillation maxima.

\section{Experimental and simulation details}

In this section we describe various experiments and the specifications used in our analysis with the summary of the experiments presented in table 1. First we give the brief descriptions of the experiments followed by details of our numerical simulations. 


\subsection{Experimental details}

\subsubsection{T2HK}

T2HK [9] is a proposed upgradation plan of the currently running T2K experiment in Japan. The neutrinos will be generated at Tokai by an upgraded version of the J-PARC beam. Currently J-PARC gives a beam power of $470 \mathrm{KW}$, but before T2HK becomes operational, the beam power will be increased beyond 1.3 MW. Although near detector of $\mathrm{T} 2 \mathrm{HK}$ is yet to be finalized, there are several ideas. Some of these are like upgradation of the current near detector ND280, building a water Čerenkov detector similar to the far detector but in a smaller scale. The far detector will be the upgradation of the currently running Super-Kamiokande (SK) [65] detector. SK is situated at $295 \mathrm{~km}$ away from Tokai at the Kamioka village and it is slightly $\left(2.5^{\circ}\right)$ off-axis to the beam-axis. Thus this will give a narrow beam centered around $0.56 \mathrm{GeV}$ which is at the first oscillation maximum of the neutrino oscillation. The upgraded SK, called Hyper-Kamiokande (HK), will consist of two identical cylindrical tanks placed upright. Each tank will contain pure water which will have a fiducial mass of $187 \mathrm{kt}$ each thus giving a total fiducial mass of $374 \mathrm{kt}$. The new detector will also have better resolution and efficiency compared to SK.

\subsubsection{T2HKK}

One of the main aim of the future long-baseline experiments is to measure the CP-violation, and T2HK is no exception. T2HK has a very good sensitivity to the CP-violation if the mass-hierarchy is known. However, the CP-violation sensitivity of T2HK drops in the unfavourable half-plane of $\delta_{C P}$ if mass-hierarchy is not known beforehand. This is due to a degeneracy between mass-hierarchy and $\delta_{C P}$ [66]. In order to solve this problem, there is a proposal to shift one of the water tanks of HK to Korea at about a distance of $1100 \mathrm{~km}$ away from the source at Tokai. This proposal is called the T2HKK [10] (the second K is for Korea.). This will have more matter effect. Thus the mass-hierarchy sensitivity will increase significantly breaking the CP-hierarchy degeneracy. However, the second detector will be at the second oscillation maximum and as the baseline is larger and the flux will also decrease.

The Korean detector site is not yet final and there are many possibilities [10]. However, all these sites are in the southern part of the Korean peninsula and lie within a range of $1-3^{\circ}$ off-axis angle with the J-PARC beam line.

\subsection{3 $\quad \mathrm{ESS} \nu \mathrm{SB}$}

$\mathrm{ESS} \nu \mathrm{SB}[11,12]$ is another future long-baseline super-beam experiment. The neutrino beam will be generated from the ESS facility at Lund, Sweden. This will have a $2 \mathrm{GeV}$ proton beam with $5 \mathrm{MW}$ beam power. The neutrino beam created by this will have a peak around $0.25 \mathrm{GeV}$. The far detector will be situated at a distance of about $540 \mathrm{~km}$ away from Lund at a mine in Garpenberg. The far detector is proposed to be $500 \mathrm{kt}$ water Cerenkov detector. At $540 \mathrm{~km}$, the second oscillation maximum occurs at $0.35 \mathrm{GeV}$. Thus the peak of the neutrino beam will be close to the second oscillation maximum for $\operatorname{ESS} \nu \mathrm{SB}$. 


\begin{tabular}{|c|c|c|c|}
\hline Experiment & Baseline $(\mathrm{L}$ in $\mathrm{km})$ & $\mathrm{L} / \mathrm{E}($ in $\mathrm{km} / \mathrm{GeV})$ & Fiducial Volume (in kton) \\
\hline T2HK & $295 \mathrm{~km}$ & 527 & $187 \times 2$ \\
\hline $\mathrm{T} 2 \mathrm{HKK}$ & $295 \mathrm{~km} ; 1100 \mathrm{~km}$ & $527(295 \mathrm{~km}) ; 1964(1100 \mathrm{~km})$ & $187(295 \mathrm{~km})+187(1100 \mathrm{~km})$ \\
\hline $\mathrm{ESS} \nu \mathrm{SB}$ & $540 \mathrm{~km}$ & 1543 & 500 \\
\hline
\end{tabular}

Table 1. The baselines, L/E and fiducial volumes of each detector for T2HK(L1), T2HK(L2) and $\mathrm{ESS} \nu \mathrm{SB}$. The energies for T2HK and $\mathrm{ESS} \nu \mathrm{SB}$ are $0.56 \mathrm{GeV}$ and $0.35 \mathrm{GeV}$ respectively.

\begin{tabular}{|c|c|c|c|}
\hline Channel & $\mathrm{T} 2 \mathrm{HK}(295 \mathrm{~km})$ & $\mathrm{T} 2 \mathrm{HK}(1100 \mathrm{~km})$ & $\mathrm{ESS} \nu \mathrm{SB}$ \\
\hline$\nu_{e}$ appearance & $3.2 \%(5 \%)$ & $3.8 \%(5 \%)$ & $3.2 \%(5 \%)$ \\
\hline$\nu_{\bar{e}}$ appearance & $3.9 \%(5 \%)$ & $4.1 \%(5 \%)$ & $3.9 \%(5 \%)$ \\
\hline$\nu_{\mu}$ disappearance & $3.6 \%(5 \%)$ & $3.8 \%(5 \%)$ & $3.6 \%(5 \%)$ \\
\hline$\nu_{\bar{\mu}}$ disappearance & $3.6 \%(5 \%)$ & $3.8 \%(5 \%)$ & $3.6 \%(5 \%)$ \\
\hline
\end{tabular}

Table 2. The signal(background) normalization uncertainties of the experiments for the various channels for T2HK, T2HKK and ESS $\nu$ SB.

\subsection{Simulation details}

We have used Global Long-Baseline Experiments Simulator (GLoBES) [67, 68] for simulating the long-baseline experiments. We present our results in terms of statistical $\chi^{2}$, where,

$$
\chi_{\text {stat }}^{2}=2 \sum_{i}\left\{N_{i}^{\text {test }}-N_{i}^{\text {true }}+N_{i}^{\text {true }} \ln \frac{N_{i}^{\text {true }}}{N_{i}^{\text {test }}}\right\},
$$

where $(N)_{i}^{\text {true }}$ corresponds to the simulated data and $(N)_{i}^{\text {test }}$ is the number of events predicted by the theoretical model. The effect of systematic errors are included by the "pull" method through the "pull" variables $\xi$. We have incorporated signal normalization error, background normalization error, signal "tilt" error \& background "tilt" error in our analysis. Incorporating the errors, the signal and background events can be written as,

$$
N_{i}^{\mathrm{test}}=\sum_{s(b)} N_{i}^{s(b)}\left(1+c_{i}^{s(b)^{\mathrm{norm}}} \xi^{s(b)^{\mathrm{norm}}}+c_{i}^{s(b)^{\mathrm{tilt}}} \xi^{s(b)^{\mathrm{tilt}}} \frac{E_{i}-\bar{E}}{E_{\max }-E_{\min }}\right)
$$

where, $s(b)$ denotes signal(background). $c_{i}^{\text {norm }}\left(c_{i}{ }^{\text {tilt }}\right)$ denotes the change in number of events by the variation of the "pull" variable $\xi^{\text {norm }}\left(\xi^{\text {tilt }}\right)$. The signal and background normalization errors are presented in table 2 . In the above equation $E_{i}$ represents the mean reconstructed energy of the $i^{\text {th }}$ bin. The maximum and minimum energy of the energy range are $E_{\min }$ and $E_{\max }$ respectively. The mean energy is given by, $\bar{E}=\left(E_{\max }+E_{\min }\right) / 2$.

The detector type proposed for T2HK, T2HKK and ESS $\nu$ SB are water Čerenkov detectors. T2HK \& T2HKK have been simulated based on [10] and the ESS $\nu$ SB experiment has been simulated according to [12]. The detector of ESS $\nu \mathrm{SB}$ is based on the MEMPHYS detector [69], which is a water Čerenkov detector. The neutrino (antineutrino) appearance channels for signal events are $\nu_{e}\left(\overline{\nu_{e}}\right)$ and the background events in this channel are 


\begin{tabular}{|c|c|c|}
\hline Oscillation parameters & True Values & Marginalization Range \\
\hline$\theta_{13}$ & $8.6^{\circ}$ & Fixed \\
\hline$\theta_{12}$ & $33.82^{\circ}$ & Fixed \\
\hline $\sin ^{2} \theta_{23}$ & $0.447(\mathrm{LO}), 0.56(\mathrm{HO})$ & $0.413-0.671$ \\
\hline$\Delta m_{21}^{2}\left(\mathrm{eV}^{2}\right)$ & $7.39 \times 10^{-5}$ & Fixed \\
\hline$\left|\Delta m_{31}^{2}\right|\left(\mathrm{eV}^{2}\right)$ & $2.52 \times 10^{-3}$ & $2.3 \times 10^{-3}-2.6 \times 10^{-3}$ \\
\hline$\delta$ & $-90^{\circ}$ & $-180^{\circ}-180^{\circ}$ \\
\hline
\end{tabular}

Table 3. The values of the 3 neutrino oscillation parameters $[1,70]$ used in the present analysis unless stated otherwise.

neutral current background, intrinsic beam background, mis-identified muons and wrongsign signal errors. The neutrino (antineutrino) disappearance channels are $\nu_{\mu}\left(\overline{\nu_{\mu}}\right)$, the background channels are neutral current background and wrong-sign muons. The signal and background normalization uncertainties have been considered as presented in table 2 . Additionally, a $5 \%$ (10\%) signal (background) "tilt" error have been considered for T2HK \& T2HKK and $0.1 \%(0.1 \%)$ in case of $\mathrm{ESS} \nu \mathrm{SB}$.

For the calculation of probability in presence of invisible neutrino decay we have modified the probability code of the GLoBES. We used $\theta_{12}=33.82^{\circ}, \theta_{13}=8.6^{\circ}$, $\sin ^{2} \theta_{23}=0.447(0.56)$ for lower (higher) octant, $\delta_{C P}=-90^{\circ}, \Delta m_{21}^{2}=7.39 \times 10^{-5} \mathrm{eV}^{2}$ and $\Delta m_{31}^{2}=2.52 \times 10^{-3} \mathrm{eV}^{2}$ for generating the simulated data (true values) as shown in the table 3 . These are consistent with the current ranges allowed by the latest global fits [1]. For the statistical studies we have marginalized over $\delta_{C P}$ and $\theta_{23}$ in their allowed ranges. We kept other standard parameters fixed as we found that they have little effects on the marginalization. We also assumed the mass hierarchy is known and it is normal hierarchy. This is because, in the case of inverted hierarchy, the heaviest state is $\nu_{2}$ instead of $\nu_{3}$. The decay of the state $\nu_{2}$ is very strongly constrained from solar neutrino data. The bounds are $\tau_{2} / m_{2}>9 \times 10^{-5} \mathrm{~s} / \mathrm{eV}$ or $\alpha_{2}<7.3 \times 10^{-12} \mathrm{eV}^{2}$. We have checked that, the experiments which we have considered are not sensitive to such low values of $\alpha_{2}$.

\section{Results}

In this section, we present the results of our study. First, we give the plots at the probability level in presence of decay for T2HK, T2HKK and ESS $\nu$ SB. These figures can be helpful in understanding many of the results presented in the next sections. Then we give the sensitivity to the decay rate $\alpha$ for each experiment. We also discuss a particular synergy which we observed between the two detectors in T2HKK experiment - one at the first oscillation maximum and one near the second oscillation maximum. We also present the results on the effect of decay on the measurement of $\theta_{23}$ and octant sensitivity for these experiments 

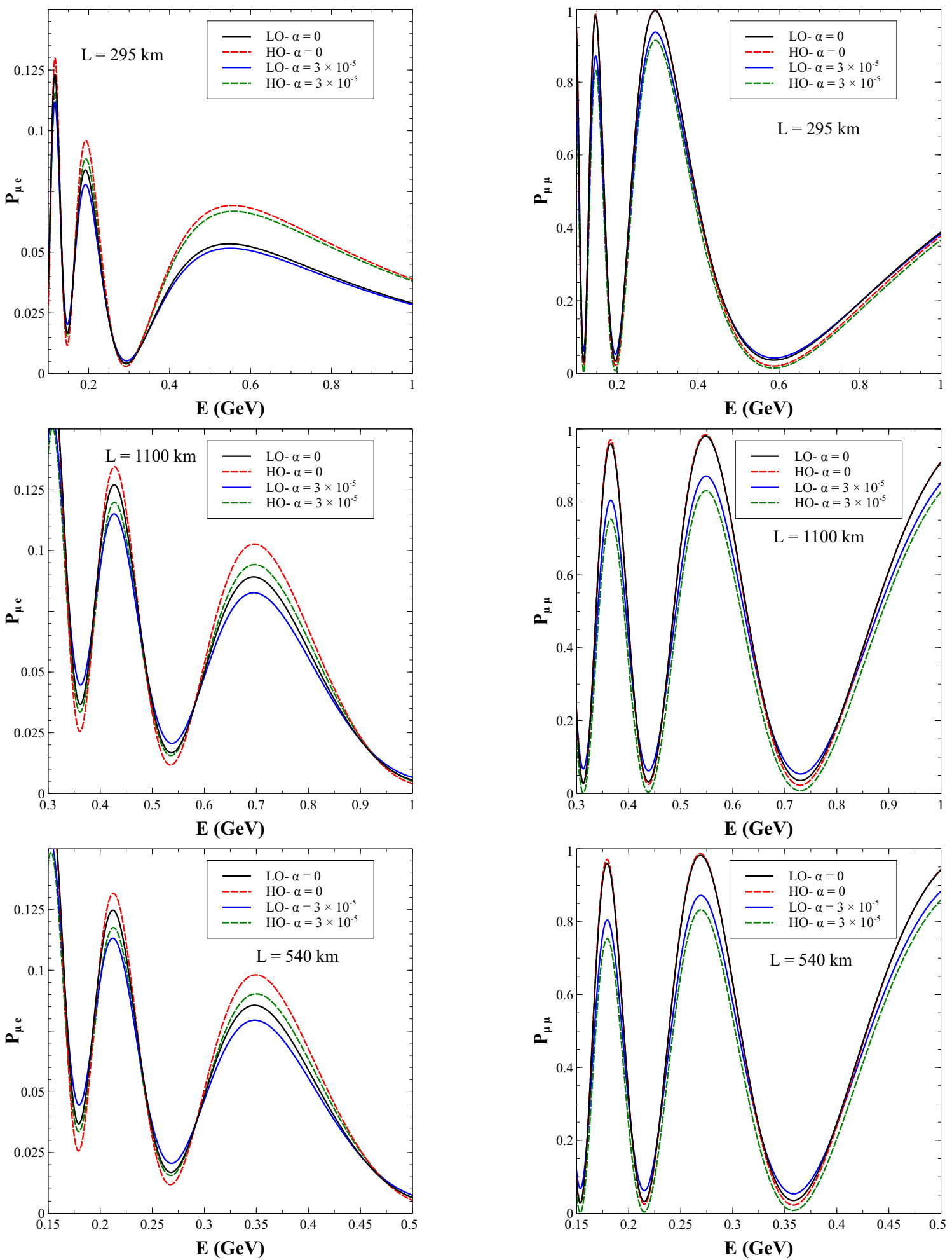

Figure 1. The probabilities as a function of energy at $\mathrm{L}=295 \mathrm{~km}, 1100 \mathrm{~km}$ and $540 \mathrm{~km}$ from top to bottom. The left (right) panel shows $\nu_{\mu} \rightarrow \nu_{e}\left(\nu_{\mu} \rightarrow \nu_{\mu}\right)$ probabilities. The black (red dashed) curves are for $\alpha=0$ and $\theta_{23}$ in the lower (higher) octant. The blue solid (green dashed) curves are for $\alpha=3 \times 10^{-5} \mathrm{eV}^{2}$ and $\theta_{23}$ in the lower (higher) octant. Where, $\theta_{23}=40^{\circ}$ for LO and $\theta_{23}=51^{\circ}$ for $\mathrm{HO}$. 


\subsection{Probability at T2HK/T2HKK and ESS $\nu$ SB baselines}

In figure 1, we present the appearance and disappearance probabilities as a function of energy for the three different baselines under investigation. We give the plots for the nodecay case $(\alpha=0)$ and also for a representative value of $\alpha=3 \times 10^{-5} \mathrm{eV}^{2}$. The choice of this value of $\alpha$ is motivated from the current bounds as obtained from $\mathrm{T} 2 \mathrm{~K}$ and $\mathrm{NO} \nu \mathrm{A}$ data analysis [38]. In both cases we give the plots for two values of $\theta_{23}$ - one in lower octant $\left(40^{\circ}\right)$ and one in the higher octant $\left(51^{\circ}\right)$. The probabilities for other values of $\theta_{23}$ within this range will lie between these two curves. The figures show the interplay between $\theta_{23}$ and $\alpha$ for the various baselines. The left (right) panel shows $\nu_{\mu} \rightarrow \nu_{e}\left(\nu_{\mu} \rightarrow \nu_{\mu}\right)$ probabilities. The black (blue) solid and red (green) dashed curves are for $\alpha=0\left(\alpha=3 \times 10^{-5} \mathrm{eV}^{2}\right)$ and $\theta_{23}$ in the lower octant and higher octant respectively. The top, middle and the bottom panel are for the baselines $295 \mathrm{~km}, 1100 \mathrm{~km}$ and $540 \mathrm{~km}$ respectively.

In the left panels of figure 1, we see that for all the baselines, at the oscillation maxima, $P_{\mu e}$ for the no decay case is higher for a fixed value of $\theta_{23}$. As decay is introduced, the probabilities reduce. On the other hand, for a fixed value of $\alpha$, lower octant gives smaller probabilities as compared to the higher octant. Thus the peak appearance probability can get reduced due to increase in the value of $\alpha$ and/or decrease in the value of the mixing angle $\theta_{23}$.

The top panel is for T2HK and here the first oscillation maximum is at $\sim 0.6 \mathrm{GeV}$ which is also where the flux peaks. For the baseline and energies involved the effect of decay for T2HK is small for the sample value of $\alpha$ considered in the plot. However, we see that the higher and lower octant bands are well separated for T2HK.

Next we focus on the middle and the bottom panels which are relevant baselines for T2HKK (second detector) and ESS $\nu$ SB. For these experiments the flux peaks near the second oscillation maxima $-\sim 0.7 \mathrm{GeV}$ for the baselines $1100 \mathrm{~km}$ and $\sim 0.35 \mathrm{GeV} 540$ $\mathrm{km}$. Since these are higher baselines and/or lower energies the effect of decay is more pronounced. The $\mathrm{HO}$ and $\mathrm{LO}$ bands are much closer in these cases and a reduction in probability due to non-zero values of $\alpha$ can be compensated by increasing $\theta_{23}$.

In the right panels of figure 1 we show the $\nu_{\mu} \rightarrow \nu_{\mu}$ disappearance probability $P_{\mu \mu}$ which plays a crucial role in determining the value of $\theta_{23}$ in long baseline experiments. In this case, the curves with $\alpha=0$ are overlapping irrespective of the octant of $\theta_{23}$ which shows that the $P_{\mu \mu}$ channel do not have any octant sensitivity in absence of neutrino decay, being dependent on $\sin ^{2} 2 \theta_{23}$. The red-dashed and black-solid curves (no-decay) are well separated from the blue-solid and green-dashed curves which reveals the effect of decay. This feature is true for all the three baselines considered in our study. In case of the 295 $\mathrm{km}$ baseline the blue curve is closer to the black and red curves compared to the $1100 \mathrm{~km}$ and $540 \mathrm{~km}$ cases since the effect of decay is less for the $295 \mathrm{~km}$ baseline for the $\alpha$ value chosen. Also, it has to be noted that, the otherwise octant degenerate $P_{\mu \mu}$ channel begins to have octant sensitivity once $\alpha \neq 0$, resulting in a gap between the blue and green curves. 

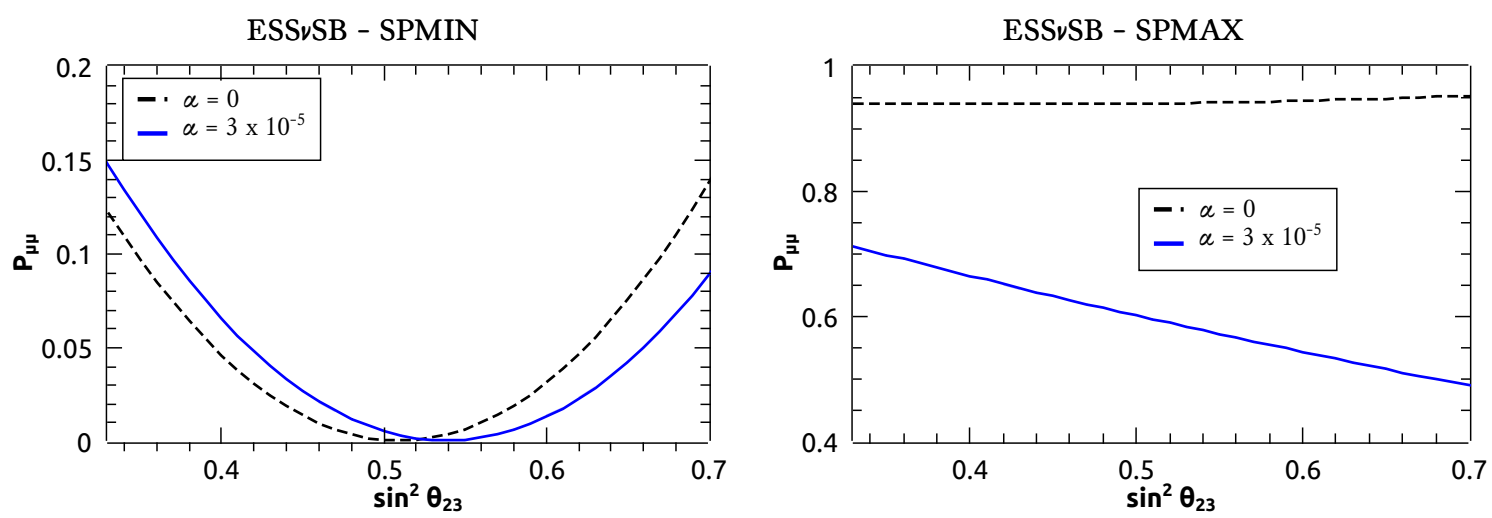

Figure 2. The probabilities as a function of $\sin ^{2} \theta_{23}$ at $\mathrm{L}=540 \mathrm{~km}$ for $\mathrm{ESS} \nu \mathrm{SB}$. The left (right) panel shows $\nu_{\mu} \rightarrow \nu_{e}\left(\nu_{\mu} \rightarrow \nu_{\mu}\right)$ probabilities. The black dashed (blue) curves are for $\alpha=0$ and $\alpha=3 \times 10^{-5} \mathrm{eV}^{2}$ respectively.

This can be simply understood if we consider the expression for two-generation survival probability in vacuum

$$
P_{\mu \mu}=\left[1-\sin ^{2} \theta_{23}\left(1-e^{-\frac{\alpha L}{E}}\right)\right]^{2}-\sin ^{2} 2 \theta_{23} e^{-\frac{\alpha L}{2 E}} \sin ^{2}\left(\frac{\Delta m_{31}^{2} L}{4 E}\right)
$$

In absence of decay the $e^{-\frac{\alpha L}{E}}$ term is 1 and the probability depends on $\sin ^{2} 2 \theta_{23}$. But in presence of decay octant sensitivity ensues due to the $e^{-\frac{\alpha L}{E}}$ factor.

For our later analysis, it is important to understand the dependence of the probability on $\theta_{23}$ at the survival probability maxima (SPMAX) and minima (SPMIN). At SPMIN, though the probability value is small, the flux peaks near this energy since it corresponds to the oscillation maxima. In figure 2 we show the behaviour of the probability for the $540 \mathrm{~km}$ baseline as a function of $\theta_{23}$ for energies corresponding to SPMAX and SPMIN. Similar behaviour is also true for the two other baselines - $295 \mathrm{~km}$ and $1100 \mathrm{~km}$. It is seen that at SPMAX, the no-decay probability is always higher than the decay probability. The no-decay probability has no dependence on $\theta_{23}$ while the decay probability reduces with increasing $\theta_{23}$ monotonically. This can be easily understood from the expression 4.1. At SPMAX, $\sin ^{2}\left(\frac{\Delta m_{31}^{2} L}{4 E}\right)=0$ and the remaining term decreases monotonically with increasing $\theta_{23}$ for non-zero $\alpha$. At SPMIN, the $\theta_{23}$ dependence is more complicated. For lower octant the decay probability is larger as compared to the no-decay probability and the probability reduces with increasing $\theta_{23}$ till a certain value (depending on $\alpha$ ), after which in higher octant it increases with $\theta_{23}$. For higher octant, no-decay probability is higher than the decay probability. As we will see, these features of the $P_{\mu \mu}$ probability will play a role in the $\chi^{2}$ fit.

\subsection{Sensitivity to the decay}

In this section we study the capability of the T2HK, T2HKK and ESS $\nu$ SB experiments in constraining invisible neutrino decay. We assume the neutrinos to be stable while generating the simulated data. In the fit we have marginalized over $\left|\Delta m_{31}^{2}\right|, \theta_{23}$ and $\delta_{C P}$ in the 

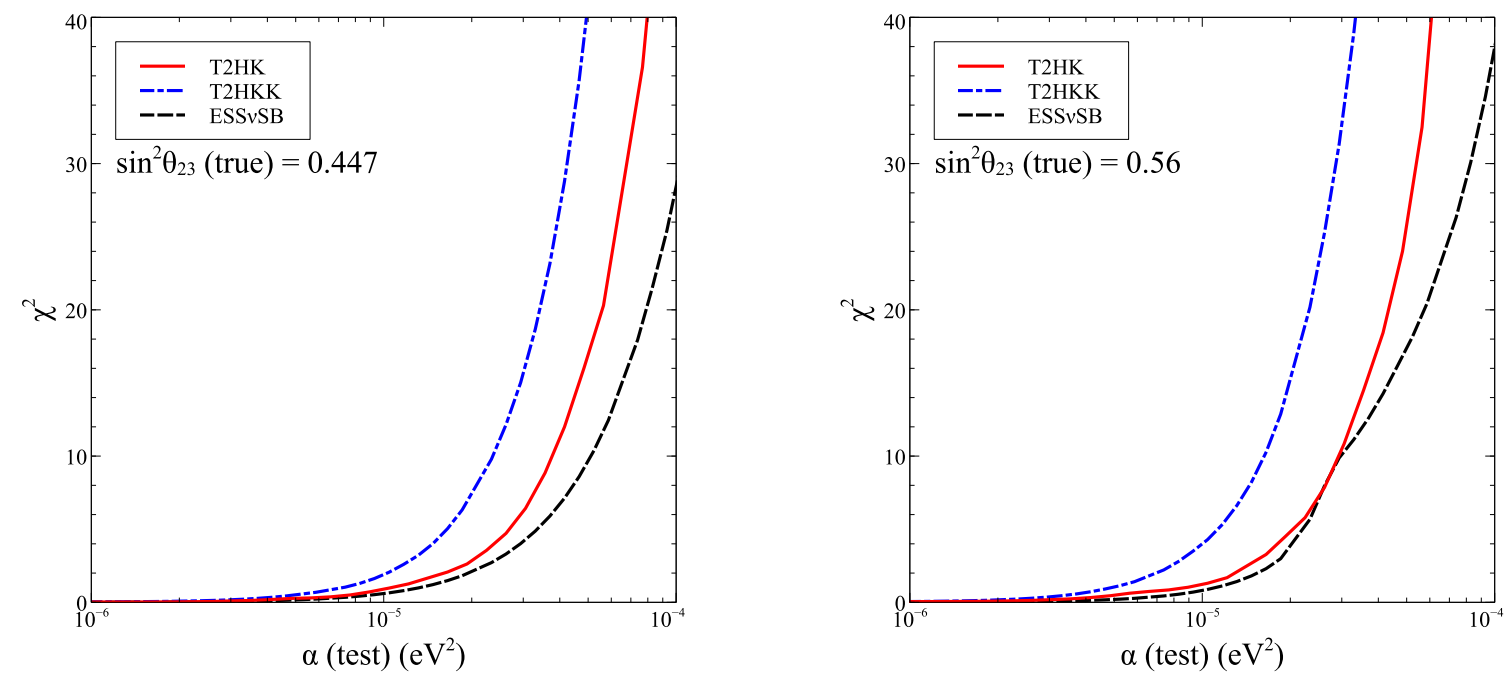

Figure 3. The $\chi^{2}$ as a function of $\alpha$, assuming no decay in the simulated data. The left (right) panel is for $\theta_{23}$ (true) in the lower (higher) octant. The red solid, blue dashed dotted and the black dashed curves are for T2HK, T2HKK and $\mathrm{ESS} \nu \mathrm{SB}$ respectively.

range given in table 3 . The figure 3 shows the value of $\chi^{2}$ for different values of $\alpha$ taken in the fit (test). A higher value of $\chi^{2}$ is more disfavoured compared to a lower values of $\chi^{2}$.

In the left panel we assumed $\sin ^{2} \theta_{23}$ (true) $=0.447$ (lower octant) while generating the data. In the right panel we assumed $\sin ^{2} \theta_{23}$ (true) $=0.56$ (higher octant). The red solid, blue dashed dotted and black dashed curves are for T2HK, T2HKK and ESS $\nu$ SB respectively. Naively, the sensitivity to decay depends on the $L / E$. From table 1 , we see that the second detector of T2HKK has highest $L / E$ and the detector at $295 \mathrm{~km}$ has lowest $L / E$ (See table 1). So, we expect T2HKK to have the best sensitivity and T2HK to have the worst, but we observe that $\mathrm{ESS} \nu \mathrm{SB}$ has a lower sensitivity than T2HK. Here, marginalization over $\theta_{23}$ plays an important role. Determination of $\theta_{23}$ is governed mainly by the $P_{\mu \mu}$ channel. In absence of decay there is no sensitivity to $\theta_{23}$ at the maxima of survival probability which corresponds to $\sin ^{2} \Delta m_{31}^{2} L / 4 E=0$. The maximum precision of $\theta_{23}$ comes at the oscillation maximum or the survival probability minimum (SPMIN) where, $\sin ^{2} \Delta m_{31}^{2} L / 4 E=1$ and can be expressed as, ${ }^{1}$

$$
\Delta\left(\sin ^{2} \theta_{23}\right)=-\frac{\Delta\left(P_{\mu \mu}\right)}{4 \cos 2 \theta_{23}}
$$

In presence of decay, the survival probability maxima also acquires some sensitivity to $\theta_{23}$ which increases with increasing value of the decay constant $\alpha$ for a fixed baseline.

As seen in the earlier section, for T2HK, the octant bands are well separated while the effect of decay is not very significant. Hence marginalization over $\theta_{23}$ does not play much role. For ESS $\nu \mathrm{SB}$ on the other hand, the octant bands are not so well separated and the effect of decay is more. As a result, marginalization over $\theta_{23}$ tends to make the probabilities

\footnotetext{
${ }^{1}$ This aspect was discussed first in [71] for the mixing angle $\theta_{12}$ in the context of solar and reactor neutrinos.
} 

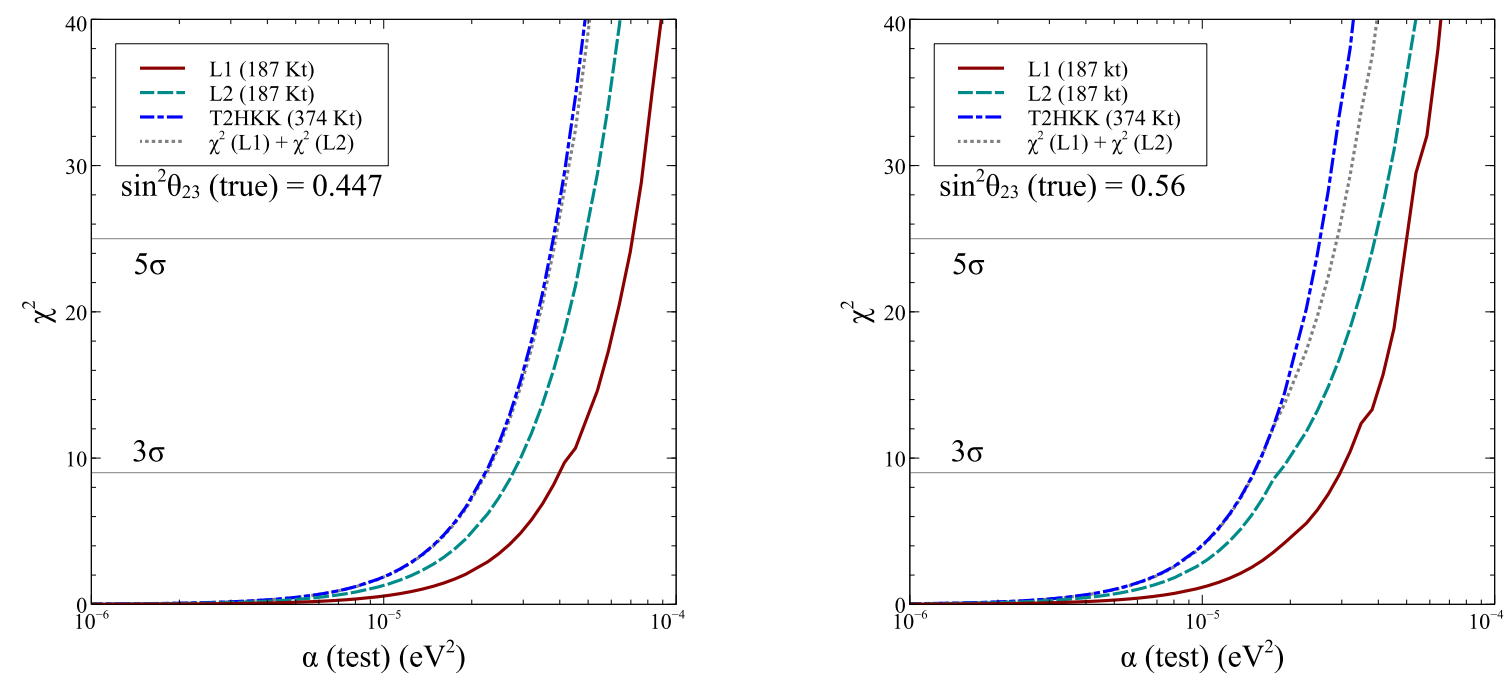

Figure 4. The $\chi^{2}$ as a function of $\alpha$, assuming no decay in the simulated data. The left (right) panel is for $\theta_{23}$ (true) in the lower (higher) octant. The dark red solid, cyan dashed, blue dashed-dotted curves are for a $187 \mathrm{kt}$ detector at $295 \mathrm{Km}, 187 \mathrm{kt}$ detector at $1100 \mathrm{~km}$ and their combination respectively. The grey dotted curve shows the $\chi^{2}$ if we just add the $\chi^{2} \mathrm{~s}$ of the dark red and cyan curves.

closer by shifting $\theta_{23}$ to a different value and the sensitivity reduces. The direction of shift depends on the initial value of $\theta_{23}$. For SPMIN, decay in the fit, can give same probability as no-decay in data for an increased $\theta_{23}$ for both lower and higher octant. This can be seen by drawing a horizontal line in figure 2. For SPMAX, on the other hand, if we fit decay with no-decay in data, then shift of $\theta_{23}$ towards lower values make the probabilities closer, giving a lower $\chi^{2}$. Thus, depending on which energy bins contribute most, the $\theta_{23}$ in the fit comes at a higher or lower value, reducing the $\chi^{2}$. We have checked that if we keep $\theta_{23}$ as fixed then $\mathrm{ESS} \nu \mathrm{SB}$ gives a better sensitivity.

In case of T2HKK, other than a detector close to the second oscillation maximum, there is also a detector at the first oscillation maximum which can measure $\theta_{23}$ with a good precision. So, the combination of the two detectors, one at the first oscillation maximum and other at the second oscillation maximum gives the best sensitivity. This will be discussed further in the next section. There is also another interesting feature for ESS $\nu \mathrm{SB}$, which is that for the true octant as higher octant we observe a sudden fall of sensitivity for $\alpha$ above $\alpha \sim 3 \times 10^{-5} \mathrm{eV}^{2}$. We will see in the next section that this is a unique feature of second oscillation maxima and we will discuss this in detail in the coming section.

\subsection{Synergy between $295 \mathrm{~km}$ and $1100 \mathrm{~km}$ baselines}

In this section we discuss a synergy between the two baselines $L 1=295 \mathrm{~km}$ and $L 2=1100$ $\mathrm{km}$ of T2HKK in constraining neutrino decay. The first one corresponds to the Japanese detector (JD) and the second one corresponds to the Korean detector (KD). It can happen that the $\chi^{2}$ obtained by combining two or more experiments can be different from the naive sum of their individual $\chi^{2}$ s. We call such enhancement of $\chi^{2}$ as synergy between the experiments. In figure 4 , we present the sensitivity to the parameter $\alpha$ for one of the 

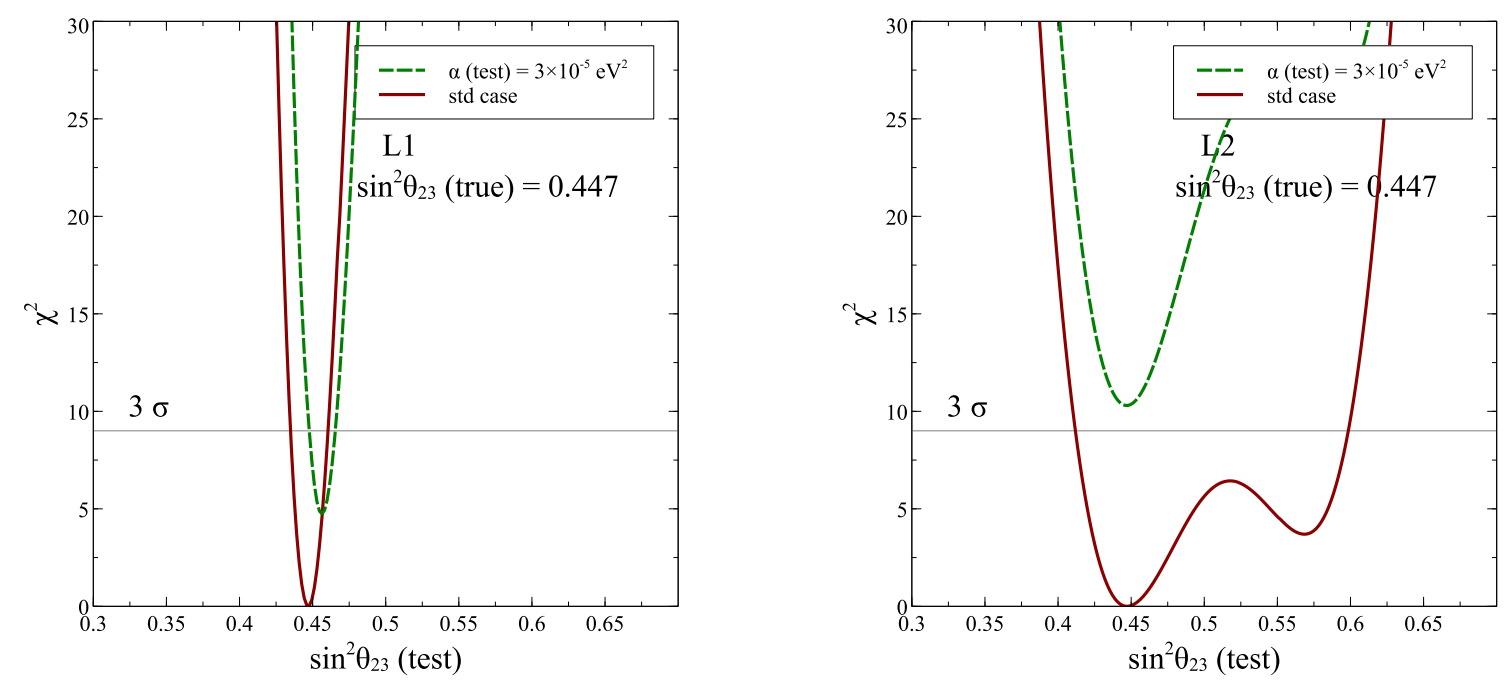

Figure 5. The $\chi^{2}$ as a function of $\theta_{23}$ assuming the true $\sin ^{2} \theta_{23}=0.447$. The left (right) panels are for baselines 295 (1100) km respectively. The dark red solid curves are for the standard cases and the green dashed curves are for the case when $\alpha=0$ in the simulated data but assumed to be $3 \times 10^{-5} \mathrm{eV}^{2}$ in the fit.

HK detectors situated at L1 (dark red solid) and L2 (cyan dashed). We also show their combined sensitivity ( blue dashed-dotted) which is nothing but the sensitivity of T2HKK. The grey dotted curve shows the naive summation of the $\chi^{2}$ s i.e $\chi_{L 1}^{2}+\chi_{L 2}^{2}$. The left panel is for $\sin ^{2} \theta_{23}=0.447$ and the right panel is for $\sin ^{2} \theta_{23}=0.56$. We see that although there is no significant synergy for the left panel, there is some significant synergy for the right panel for $\alpha$ greater than $2.268 \times 10^{-5} \mathrm{eV}^{2}$, i.e., the $\chi_{L 1+L 2}^{2}$ of T2HKK is larger than the $\chi_{L 1}^{2}+\chi_{L 2}^{2}$.

This synergy can be understood from the figure 5 and figure 6 . These figures give the $\chi^{2}$ for a given value of $\theta_{23}$ in the fit with a fixed value of $\alpha$ in the fit. The decay constant $\alpha=0$ while simulating the data and $\left|\Delta m_{31}^{2}\right|$ and $\delta_{C P}$ are marginalized for all the cases. Therefore global minima of the curves in these figures give the $\chi^{2}$ values for the corresponding $\alpha$ (test) in figure 4. Figure 5 is for the case when $\sin ^{2} \theta_{23}=0.447$ and figure 6 is for $\sin ^{2} \theta_{23}=0.56$ in the simulated data. The dark red solid curves assume $\alpha=0$ in the fit and green dashed curves assume $\alpha=3 \times 10^{-5} \mathrm{eV}^{2}$ in the fit.

First let us consider the figure 5. Here, $\sin ^{2} \theta_{23}($ true $)=0.447$. We see that for L1, the global minimum, for a test value of $\alpha=3 \times 10^{-5} \mathrm{eV}^{2}$ in the fit, shifts slightly towards a higher $\sin ^{2} \theta_{23}$ but stays in the same octant as the $\alpha=0$. The shift towards a higher value can be understood by looking at the curve for $P_{\mu \mu}$. It is seen that at the oscillation maxima (i.e SPMIN), the no-decay probability (black) curve is lower than that with decay (blue curve) for lower octant. Hence when we try to fit data with no-decay with decay in theory, the probabilities can come closer by increasing $\theta_{23}$ slightly. Similar feature is also observed at L2 in the right panel. the shift is almost un-noticeable. Sensitivity to the parameter $\alpha$ for the combination L1 and L2 will be given by the global minimum of the sum of $\chi^{2} \mathrm{~s}$ of the two green dashed curves of figure 5. As the global minima of these two curves approximately coincide $\left(42^{\circ}\right.$ for L2 and $42.5^{\circ}$ for L1), the global minimum of the sum of 

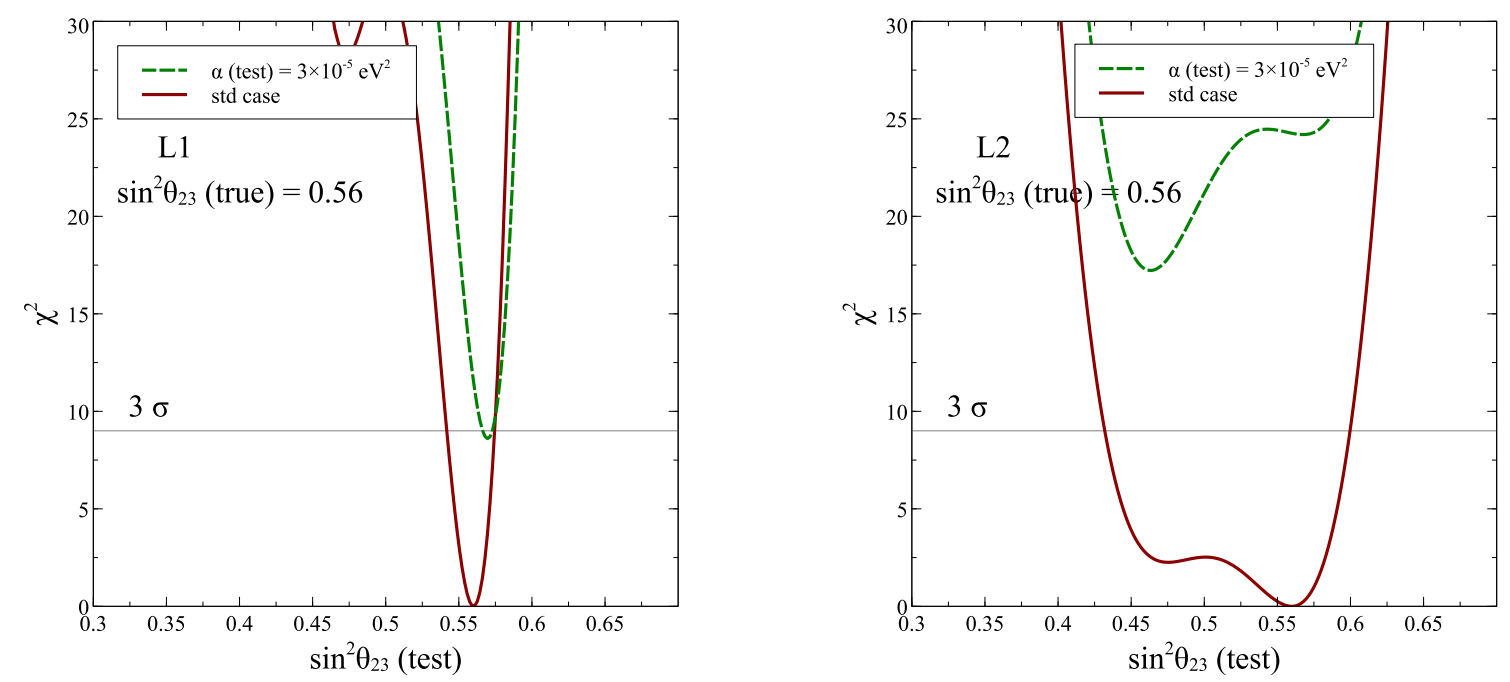

Figure 6. The $\chi^{2}$ as a function of $\theta_{23}$ assuming true $\sin ^{2} \theta_{23}=0.56$. The left (right) panels are for baselines 295 (1100) $\mathrm{km}$ respectively. The dark red solid curves are for the standard cases and the green dashed curves are for the case when $\alpha=0$ in the simulated data but assumed to be $3 \times 10^{-5} \mathrm{eV}^{2}$ in the fit.

their $\chi^{2} \mathrm{~s}$ will be approximately equal to the sum of $\chi^{2} \mathrm{~s}$ at the global minima of L1 and L2. Therefore, the sensitivity to the combination of L1 and L2 will be approximately equal to the sum of the sensitivities for L1 and L2. Thus there is not significant synergy for this case.

Next we consider the figure 6 . Here, $\sin ^{2} \theta_{23}=0.56$. We see that for $\mathrm{L} 1$, like previous case, the global minimum for $\alpha=3 \times 10^{-5} \mathrm{eV}^{2}$ (test) shifts a little towards higher value since at SPMIN, no-decay probability is larger, it can be matched by increased value of $\theta_{23}$ (cf. 2).

However, for L2, we see that the position of the global minimum drastically changes and now it is in the opposite octant, i.e., lower octant. Again, like the previous case, the sensitivity to the combination of L1 and L2 will be determined by the sum of $\chi^{2} \mathrm{~s}$ of two green dashed curves. But here, the positions of the global minima for L1 and L2 are completely different. Therefore, the position of the global minimum of the sum of $\chi^{2} \mathrm{~s}$ of L1 and L2 curves is non-trivial and is not equal to the sum of the $\chi^{2} \mathrm{~s}$ at the global minima of L1 and L2 curves. Hence, we see the synergy in this case. The shift of the global minimum can be attributed to the interplay between the appearance and disappearance channels.

In order to understand this interplay in figure 7 we have plotted the appearance and disappearance $\chi^{2} \mathrm{~s}$ for two different values of the decay constant $\alpha$ for L2. From the left panel of the figure it is seen that for true $\sin ^{2} \theta_{23}$ in the lower octant, the minima for both appearance and disappearance channels stay in the correct octant. The octant sensitivity is seen to come from both disappearance and appearance channel. However, for appearance channels, the octant bands are not very widely separated for experiments close to second oscillation maxima, and with increase in $\alpha$ the effect of disappearance channel becomes more pronounced and there is a sharp rise in the octant sensitivity coming from the disappearance channel. 
T2HKK (L2)

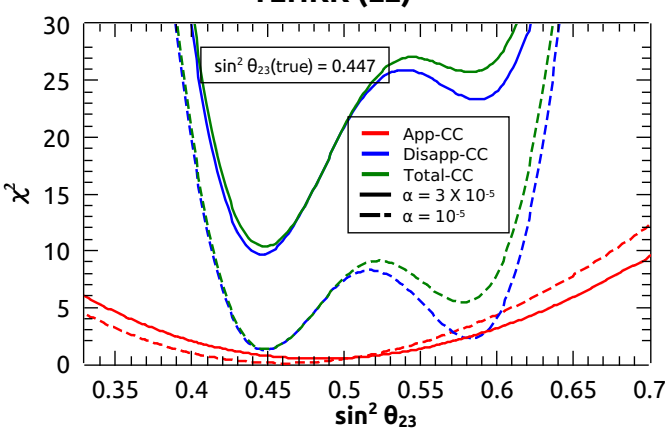

T2HKK (L2)

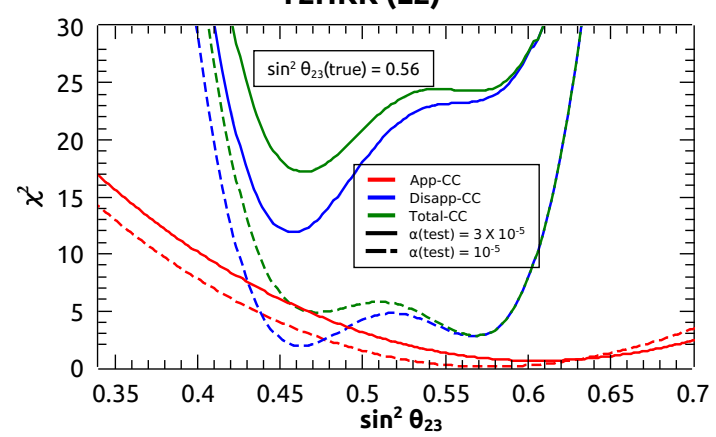

Figure 7. The $\chi^{2}$ as a function of $\theta_{23}$. The left (right) panels are for true $\sin ^{2} \theta_{23}=0.447$ $\left(\sin ^{2} \theta_{23}=0.56\right)$ respectively. The red and blue curve are for appearance and disappearance channels respectively. While the total contribution from both the channels is represented by green curves. The solid curves are for $\alpha$ (test) $=3 \times 10^{-5} \mathrm{eV}^{2}$ and the dashed curves for $\alpha$ (test) $=10^{-5} \mathrm{eV}^{2}$ in the fit. The data have been generated for $\alpha=0$.

The right panel depicts the situation for the higher octant. In this case when the no-decay in data is fitted with decay, the minima for the appearance channel stays in the same octant, shifting slightly towards higher $\theta_{23}$. This behaviour can be understood from the appearance probability since for this case, $\mathrm{HO}$ and no-decay gives a higher probability and hence if we fit with decay, the $\theta_{23}$ tends to shift to a larger value to get closer to the data. As the decay constant increases, the shift in the value of $\theta_{23}$ to get closer to the no-decay curve will be higher.

On the other hand the disappearance minima is seen to shift to the lower octant. For a lower value of the decay constant, the difference between the disappearance $\chi^{2}$ minimum in the two octants is not very high. On the other hand, the appearance $\chi^{2}$ rises steeply in the opposite octant and when the appearance $\chi^{2}$ is added to the disappearance $\chi^{2}$, the overall minima comes in the correct octant. But for a higher value of the decay constant, the minima in the wrong octant is much lower than the minima in the correct octant and thus the overall minima comes in the wrong octant, being driven by the disappearance channel. The reason for the disappearance channel preferring the lower octant can be understood from the probability figure 1 , by noticing that for peak flux energies of around $0.6 \mathrm{GeV}$, the red-curves (no-decay, HO) are closer to the blue curves (decay, LO). Hence, when data is generated in the higher octant with no decay, disappearance channel prefers the lower octant if $\alpha \neq 0$ in the fit, the shift being higher as the decay constant increases. This effect is not present at the detector tuned for the first oscillation maximum at $295 \mathrm{~km}$. From the top-left panel of figure 1, we see that the octant bands are wide enough such that the appearance channel gives high octant sensitivity and therefore the appearance channel always dominates and we do not get any false octant solution.

The above discussion can be now used to explain the kink in the sensitivity of ESS $\nu \mathrm{SB}$ (figure 3 right panel). For ESS $\nu$ SB there is only a single detector. For lower values of $\alpha$, the disappearance channel is still weak and the appearance channel dominates and we get the octant in the higher octant. However, as the $\alpha$ becomes more than some critical value, 

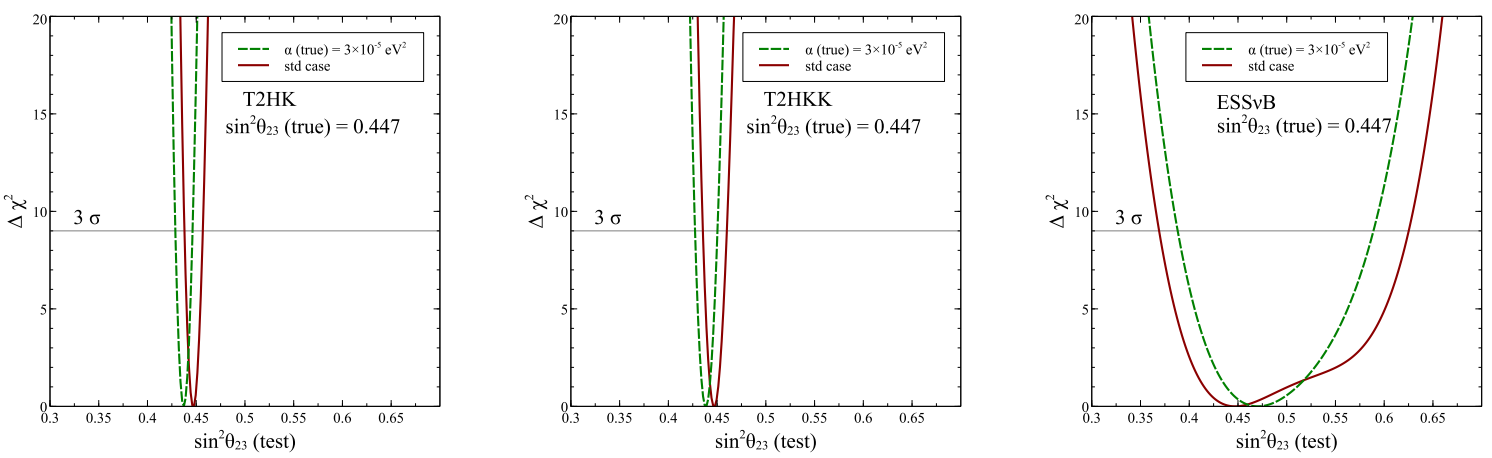

Figure 8. The $\Delta \chi^{2}$ as a function of $\theta_{23}$ (test) assuming $\sin ^{2} \theta_{23}$ (true) $=0.447$. The left, middle and the right panels are for T2HK, T2HKK and ESS $\nu$ SB respectively. The dark red solid curves are for standard cases and the green dashed curves are for the cases where $\alpha$ is assumed to be $3 \times 10^{-5} \mathrm{eV}^{2}$ in the simulated data but decay is not considered in the fit.

the disappearance channel begins to dominate and the octant flips to the wrong side. Thus the $\chi^{2}$ is abruptly decreased, giving a lower sensitivity.

\subsection{Role of decay in the $\theta_{23}$ determination}

In this section, we will see how presence of invisible neutrino decay can affect the measurement of $\theta_{23}$ in T2HK, T2HKK and $\mathrm{ESS} \nu \mathrm{SB}$ experiments.

Figure 8 gives $\Delta \chi^{2}$ as a function of $\theta_{23}$ (test). Here, we assumed $\sin ^{2} \theta_{23}=0.447$ in the data. The left, middle and right panels are for T2HK, T2HKK and ESS $\nu$ SB respectively. In the fit, we marginalized over, $\left|\Delta m_{31}^{2}\right|, \delta_{C P}$ and kept $\alpha$ fixed at zero. For dark-red solid curves, we assumed stable neutrino in the data and for green dashed curves, we assumed $\alpha=3 \times 10^{-5} \mathrm{eV}^{2}$ in the data.

We see that for the three cases, the best-fit values for data generated for $\alpha=3 \times$ $10^{-5} \mathrm{eV}^{2}$ and $\alpha=0$ are different. We notice that for true $\alpha=3 \times 10^{-5} \mathrm{eV}^{2}$ the best-fit of $\theta_{23}$ is shifted towards lower values for T2HK and T2HKK. However, for ESS $\nu$ SB, the shift is in the opposite direction. The shift towards lower values for T2HK and T2HKK is governed by the behaviour of $P_{\mu \mu}$ at the oscillation maxima i.e at SPMIN, where the flux peaks. The data is generated for decay which gives a higher probability than no-decay in the lower octant. Therefore when data is fitted with $\alpha=0$, a reduced value of $\theta_{23}$ gives a better fit since the probability increases with decreasing $\theta_{23}$.

The reason for different behaviour for $\mathrm{ESS} \nu \mathrm{SB}$ is more complicated. Here, resolution plays a big role. The resolution of the detector smears the imprint of probability over all energy bins. As a result, the feature near the oscillation minima is lost due to the larger bin width of $0.1 \mathrm{GeV}$. A bin from $0.3 \mathrm{GeV}$ to $0.4 \mathrm{GeV}$ contains events from the survival probability minima as well as the features of the probability around $0.3 \mathrm{GeV}$ where the probabilities corresponding to decay and no decay curves show opposite behaviour than at $\sim 0.35 \mathrm{GeV}$. As an upshot if one plots the events then the no-decay events are higher than the events with decay in most bins. When the simulated event spectrum is fitted without decay, $\theta_{23}$ is shifted towards higher value to bring the probability down. The 

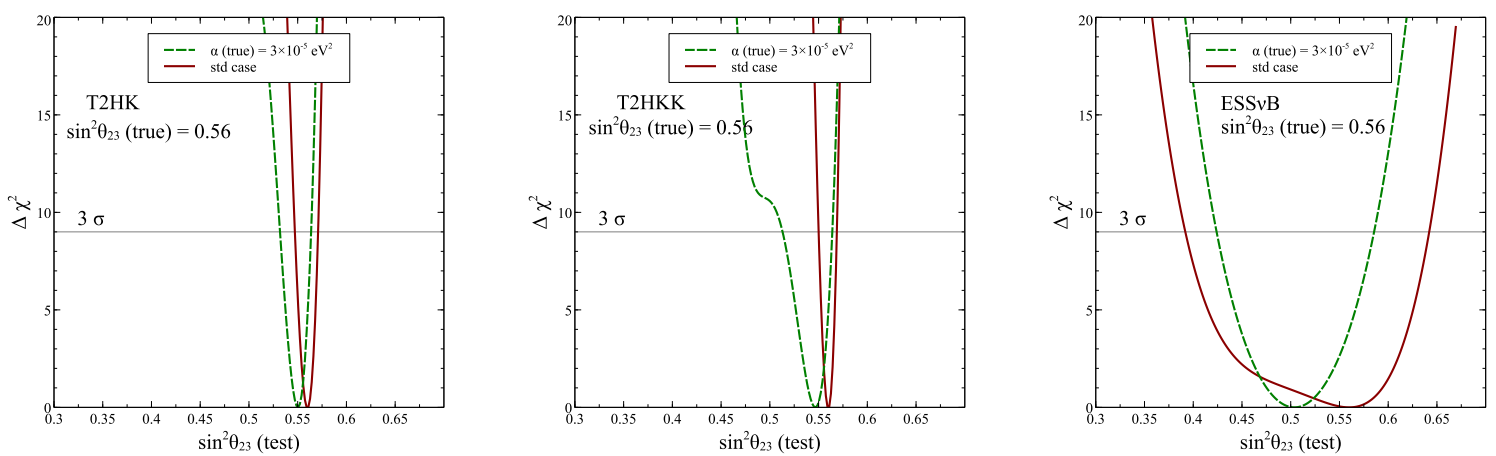

Figure 9. The $\Delta \chi^{2}$ as a function of $\theta_{23}$ (test) assuming $\sin ^{2} \theta_{23}$ (true) $=0.56$. The left, middle and the right panels are for T2HK, T2HKK and ESS $\nu \mathrm{SB}$ respectively. The dark red solid curves are for standard cases and the green dashed curves are for the cases where $\alpha$ is assumed to be $3 \times 10^{-5} \mathrm{eV}^{2}$ in the simulated data but decay is not considered in the fit.

effect of smearing affects the experiments close to second oscillation maxima more since the variation with probability is sharper compared to the first oscillation maxima.

Figure 9 is similar to figure 8 , but here, we assume $\sin ^{2} \theta_{23}=0.56$ in the data. We see that, like the previous figure, the best-fit values of $\theta_{23}$ is changed but in this case, for all three experiments, the shifts of $\theta_{23}$ are towards a lower value. This can be explained since, for $\theta_{23}$ in the higher octant the probability for non-zero $\alpha$ is smaller than the probability with $\alpha=0$ for both SPMIN and SPMAX and hence no-decay gives larger number of events. For $\theta_{23}$ in higher-octant, lowering $\theta_{23}$ reduces the probability and hence gives a better fit when we fit decay in data with no-decay.

In figure 10, we show the octant sensitivities as a function of the CP phase $\delta_{C P}$ for experiments which are tuned to the second oscillation maxima in presence and absence of decay. The top panels are for ESS $\nu \mathrm{SB}$ and the bottom panels are for a hypothetical T2HKK experiment whose both detectors are at $1100 \mathrm{~km}$. The left panels are for no decay and the right panels are for the case when $\alpha \neq 0$. The red lines are for appearance channels, the blue lines are for disappearance channels and the green lines are for both appearance and disappearance channels together. In absence of decay disappearance channel does not have any octant sensitivity. However when appearance $\chi^{2}$ is added to disappearance $\chi^{2}$, then the appearance probability being dependent on $\sin ^{2} \theta_{23}$ rises monotonically in the opposite octant and gives a large octant-sensitive contribution. In presence of decay, the disappearance channel itself has octant sensitivity, which enhances the overall octant sensitivity.

\subsection{Combined analysis}

Here in figure 11, we have shown sensitivities of different experiments to constrain the decay parameter $\alpha$ (test) with (left) and without (right) decay in 'data' assuming the higher octant as the true octant. The plots in the left panel show how precisely these experiments can measure $\alpha$ and hence for a given true value how they can exclude the no decay scenarios. On the other hand, plots in the right panel show the constraints these experiments can put on invisible neutrino decay. The results are shown for all the three experiments ESS $\nu \mathrm{SB}$, 

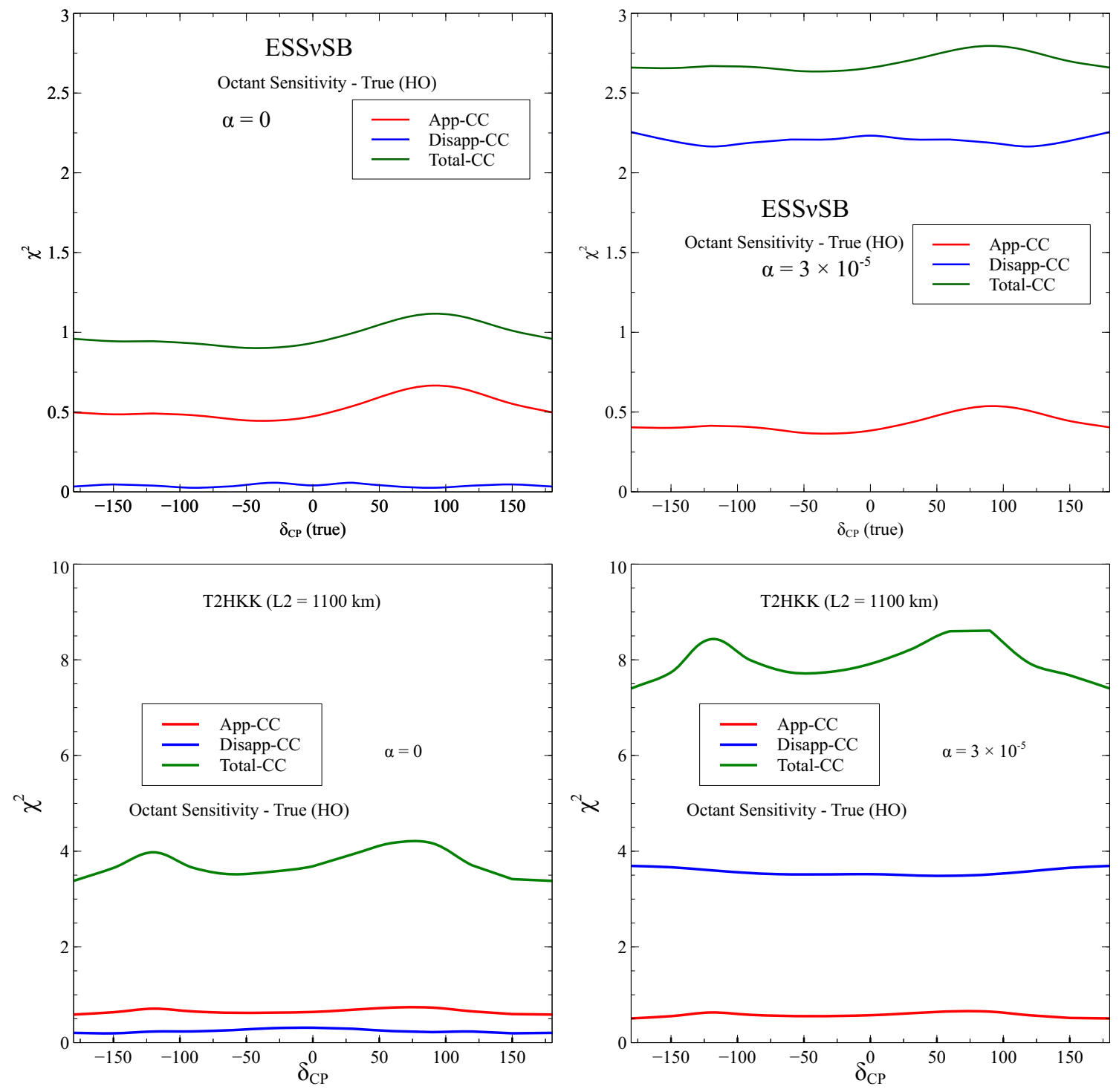

Figure 10. The octant sensitivity $\chi^{2}$ as a function of $\delta_{C P}$ (true) for $\operatorname{ESS} \nu \operatorname{SB}$ (top row) and T2HKKL2 (bottom row). The left panel represents the octant sensitivity for the case without decay i.e. $\alpha=0$ and the right panel for the case with decay $\alpha=3 \times 10^{-5}$. The $\alpha$ values are same for both data and fit in each plot. The red and blue plots are for appearance-CC, disappearance-CC respectively, while the green plots shows the contribution from both appearance-CC and disappearance-CC. The true $\sin ^{2} \theta_{23}=0.56$ and the test $\theta_{23}$ is varied in the lower octant in the range $40^{\circ}<\theta_{23}<45^{\circ}$. 

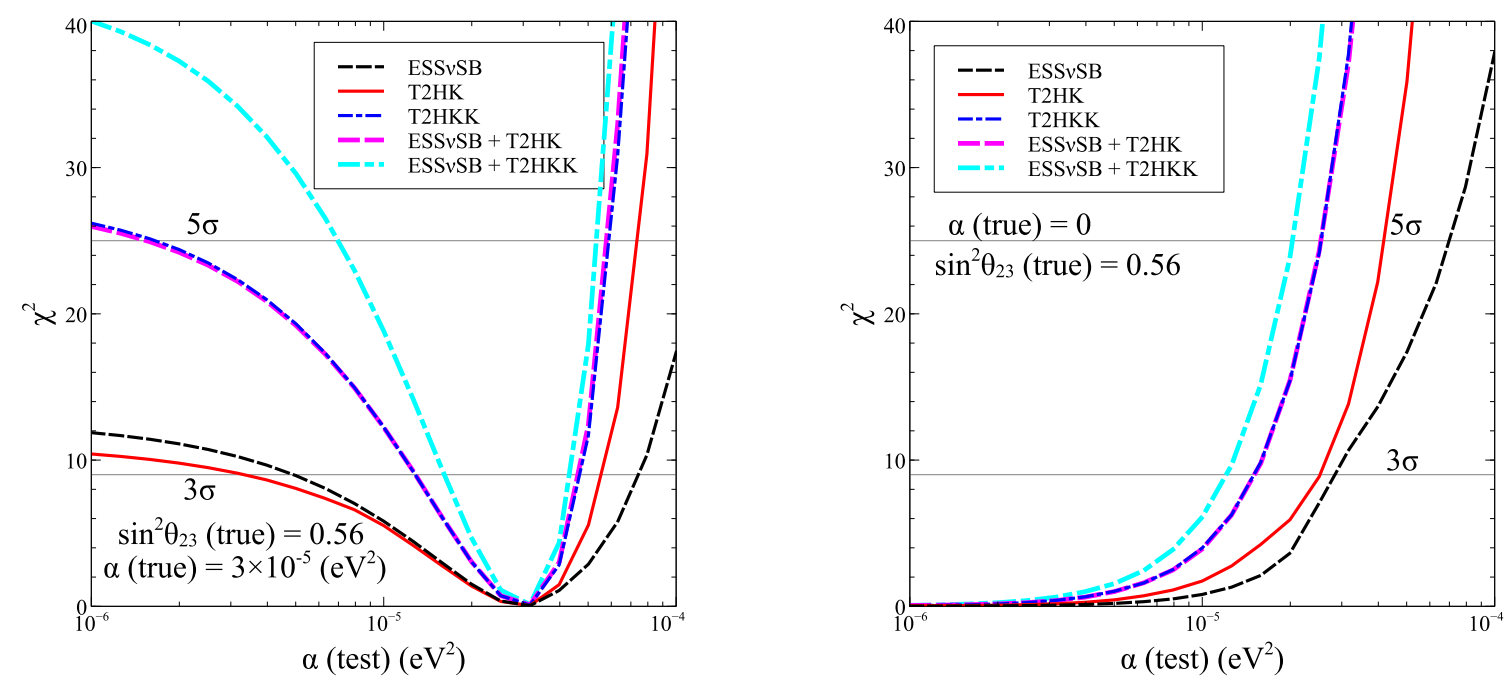

Figure 11. The $\chi^{2}$ as a function of test $\alpha$ for various combination of experiments. The left panel is for the case where $\alpha=3 \times 10^{-5}\left(\mathrm{eV}^{2}\right)$ is assumed for the data and the right panel is for the case where $\alpha=0$ in the decay. The black dashed curves are for $\operatorname{ESS} \nu \mathrm{SB}$, red solid curves are for T2HK, blue dashed-dotted curves for T2HKK, the magenta dashed for combination of ESS $\nu \mathrm{SB}$ and T2HK and the cyan curves are for combination of $\operatorname{ESS} \nu \mathrm{SB}$ and T2HKK.

T2HK, T2HKK and the combination of ESS $\nu$ SB with both T2HK and T2HKK. In the figure 11, the black dashed line shows the sensitivity of $\mathrm{ESS} \nu \mathrm{SB}$, the blue dashed-dotted line is for T2HKK, while the red solid line represent the same for T2HK. The pink and cyan dashed line stands for the combination of $\mathrm{ESS} \nu \mathrm{SB}$ with T2HK and T2HKK respectively.

It is observed from the left panel where we have assumed decay in the simulated data, that T2HKK has the highest precision out of the three and at $3 \sigma$ it can precisely measure $\alpha$ in the range $1.278 \times 10^{-5}<\alpha<4.614 \times 10^{-5} \mathrm{eV}^{2}$ for the given true value of $\alpha=3 \times 10^{-5} \mathrm{eV}^{2}$. For the same true value of $\alpha \mathrm{T} 2 \mathrm{HK}$ gives better precision than $\mathrm{ESS} \nu \mathrm{SB}$ and at $3 \sigma$ the range is $3.408 \times 10^{-6}<\alpha<5.524 \times 10^{-5} \mathrm{eV}^{2}$ and $4.945 \times 10^{-6}<\alpha<7.303 \times 10^{-5} \mathrm{eV}^{2}$ respectively. Combining T2HKK with ESS $\nu$ SB improves the bounds further and at $3 \sigma$ the range is $1.597 \times 10^{-5}<\alpha<4.231 \times 10^{-5} \mathrm{eV}^{2}$. Although the combination of T2HK and ESS $\nu \mathrm{SB}$ improves the bound, still it comes to be similar to that of T2HKK. In the right panel of figure 11, we observe that by combining two experiments it is possible to improve the constraint on $\alpha$. Specially, the combination of T2HKK and ESS $\nu$ SB gives the highest limit on $\alpha$ and at $3 \sigma$ the constraint is $\alpha \leq 1.193 \times 10^{-5} \mathrm{eV}^{2}$. Similarly, T2HK+ ESS $\nu \mathrm{SB}$ also improves the constrain and the derived limit in this case is very much equal to that of T2HKK.

In figure 12 we present two complementary plots to figure 11. If neutrino decays in nature in addition to oscillation, then how it affects the measurements of $\theta_{23}$ is shown in the left panel of figure 12 . We have shown the results in the $\theta_{23}$ (test)- $\alpha$ (test) parameter space at $95 \%$ C.L. We observe that the parameter space shrinks noticeably when we combine two experiments. Again in the right panel, we show the allowed region in $\theta_{23}$ (test)$\alpha$ (test) parameter space assuming no decay scenario in the simulated data. Here also, the combination of experiments gives better results that the individual experiments. 

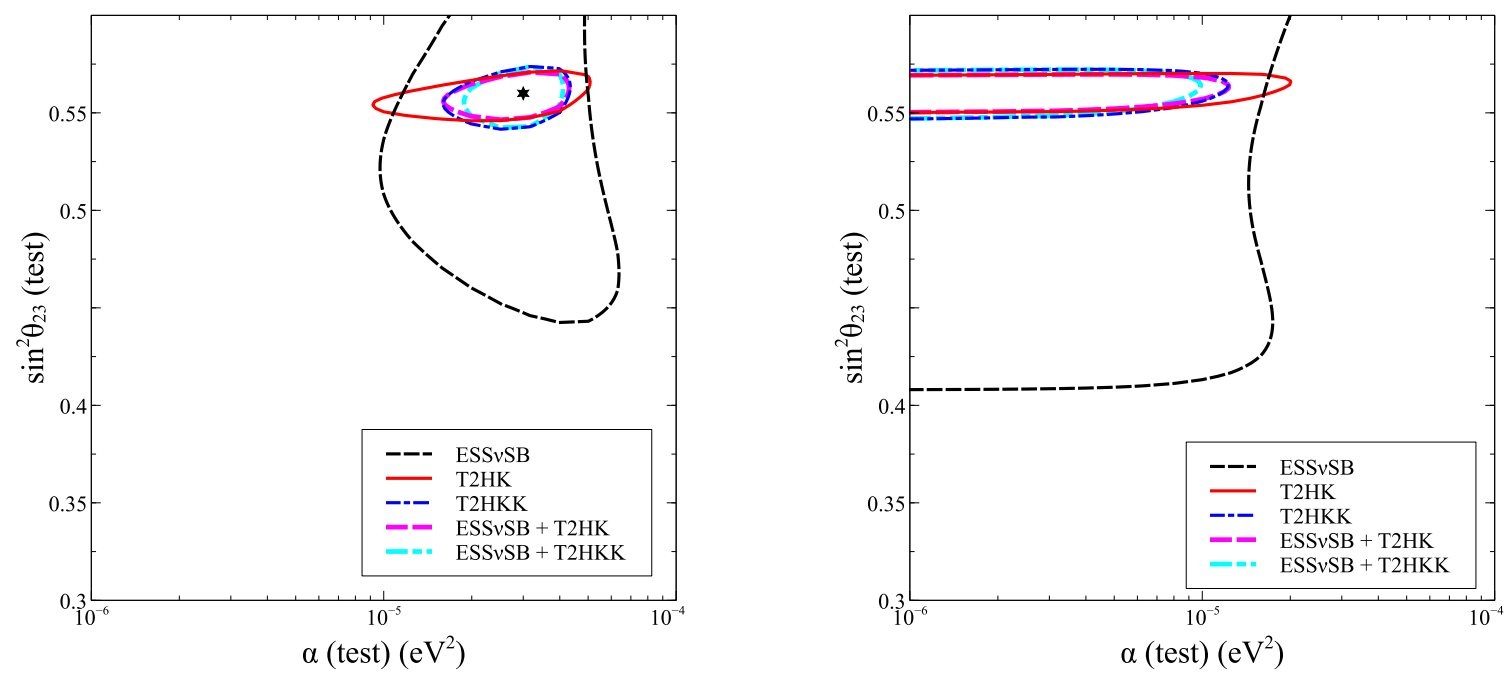

Figure 12. The $95 \%$ confidence contours in the $\theta_{23}-\alpha$ plane for various combination of experiments. The left panel is for the case where $\alpha=3 \times 10^{-5}\left(\mathrm{eV}^{2}\right)$ is assumed for the data and the right panel is for the case where $\alpha=0$ in the decay. The black dashed curves are for $\mathrm{ESS} \nu \mathrm{SB}$, red solid curves are for T2HK, blue dashed-dotted curves for T2HKK, the magenta dashed for combination of $\mathrm{ESS} \nu \mathrm{SB}$ and T2HK and the cyan curves are for combination of $\mathrm{ESS} \nu \mathrm{SB}$ and T2HKK.

\section{$5 \quad$ Summary \& conclusions}

We have examined the physics potential of future long-baseline experiments T2HK/T2HKK and $\mathrm{ESS} \nu \mathrm{SB}$ in the context of invisible neutrino decay. We performed our study for the case of normal hierarchy where $\nu_{3}$ is the highest mass state and this is assumed to be unstable, decaying into lighter sterile states. We compared and contrasted the sensitivities to decay of these experiments with special emphasis on the location of the experiments at first and second oscillation maximum and the various factors that can affect the sensitivities.

The effect of decay appears as $\exp (-\alpha L / E)$ where, $\alpha$ is the decay constant, $L$ is the distance traveled and $E$ is the energy. Hence, it is expected that effect of decay will be more pronounced for second oscillation maxima since this occurs at lower energy for same baseline or higher baseline for same energy. However, the experimental sensitivities for the decay rate also depend on other vital factors like neutrino flux, resolution of the detector and the sensitivity of the experiment to resolve $\theta_{23}$. We expounded in detail which factors play important roles in determining the sensitivity to decay at the first and second oscillation maximum. Among the three experiments T2HK is designed to have its flux peak at the first oscillation maxima, T2HKK has one detector at the first oscillation maximum and one near the second oscillation maximum, while $\mathrm{ESS} \nu \mathrm{SB}$ is an experiment at the second oscillation maximum. T2HK has a lower L/E as compared to the other experiments and has less sensitivity to decay. On the other hand being at the first oscillation maxima it has a better sensitivity to $\theta_{23}$ and its octant. In comparison, ESS $\nu \mathrm{SB}$ has a higher L/E, but because of lower energy resolution as well as poor sensitivity to $\theta_{23}$, it has a reduced sensitivity to the decay constant $\alpha$. The two detector set up of T2HKK helps since the detector at the first oscillation maximum helps in determining $\theta_{23}$ while the second helps 
in better constraining $\alpha$. We have studied the sensitivity of these experiments to the decay rate $\alpha$ for true $\theta_{23}$ in both lower and higher octant.

We have also presented the octant sensitivity as a function of $\delta_{C P}$ for the three experiments. We have obtained an interesting result that in presence of decay, the overall octant sensitivity is enhanced. This can be attributed to the octant sensitive contribution coming from the disappearance channel in presence of decay. Since T2HK is at the first oscillation maxima, the appearance channel already gives a good sensitivity and the effect of disappearance channel is not very significant. But since $\mathrm{ESS} \nu \mathrm{SB}$ is at the second oscillation maximum the octant sensitivity coming from appearance channel is not very high and the disappearance channel plays a consequential role in enhancing the octant sensitivity in presence of decay. Similar feature is also observed for the detector at $1100 \mathrm{~km}$ baseline with flux peak near the second oscillation maxima.

The octant sensitivity coming from the disappearance channel increases with the decay constant $\alpha$ and also leads to an interesting interplay with the appearance channel in determining the overall minimum for experiments at second oscillation maxima. At the first oscillation maxima on the other hand, the appearance channel dominates. As a result of this different behaviour, for T2HKK, which has one detector at first and one at second oscillation maxima, a synergy is observed for true $\theta_{23}$ in the higher octant leading to a $\chi^{2}$ greater than the naive sum of the $\chi^{2} \mathrm{~s}$ at two different baselines. Since at second oscillation maximum the appearance channel does not have very good octant sensitivity the $\chi^{2}$ minimum for the second detector comes in the wrong octant driven by the disappearance channel. The $\chi^{2}$ minima for the detector at the first oscillation maximum on the other hand stays in the correct octant (the wrong octant solution being disfavoured by the appearance channel). As an upshot, the global minima while combining the two baselines come in a position different from the individual minima resulting in the synergy. On the other hand, for ESS $\nu \mathrm{SB}$, the first detector is not present and the leaning of the disappearance channel towards the wrong octant, weaken the sensitivity beyond a certain value of the decay constant $\alpha$.

Given the correlation between $\alpha$ and $\theta_{23}$, the determination of $\theta_{23}$ can also get affected, if there is decay in nature but it is ignored in the fit resulting in a wrong determination of $\theta_{23}$. We have examined this issue in detail and found that the shift of $\theta_{23}$ from its true value, can be in either direction, depending on the octant of true $\theta_{23}$ and whether the contribution is coming from bins centered at the maxima or minima of the survival probability, which in turn depends on the smearing and bin-width. This effect is more for the second oscillation maxima experiments since the probability band is narrower.

Apart from the sensitivity study for individual experiments we have also performed sensitivity studies for the two combinations of T2HK $+\mathrm{ESS} \nu \mathrm{SB}$ and T2HKK $+\mathrm{ESS} \nu \mathrm{SB}$. We found that the sensitivity increases significantly if we combine the experiments. The sensitivity to the decay-rate attained by the experiments T2HK, T2HKK, ESS $\nu$ SB and their combinations of $\mathrm{T} 2 \mathrm{HK}+\mathrm{ESS} \nu \mathrm{SB}, \mathrm{T} 2 \mathrm{HKK}+\mathrm{ESS} \nu \mathrm{SB}$ have been summarized in the table 4.

We have also done a precision study assuming $\alpha=3 \times 10^{-5} \mathrm{eV}^{2}$ in the data and found that the precision is maximum for the combination of $\mathrm{T} 2 \mathrm{HKK}+\mathrm{ESS} \nu \mathrm{SB}$ while standalone T2HKK has the best precision. This also reiterates the fact that combination of first and second oscillation maximum can give the best sensitivity to decay. Although combination of the experiments improve the sensitivity to $\alpha$, no significant improvement is observed for $\theta_{23}$. 


\begin{tabular}{|c|c|c|}
\hline Experiment(s) & $\alpha\left(\mathrm{eV}^{2}\right)$ & $\tau_{3} / m_{3}(\mathrm{~s} / \mathrm{eV})$ \\
\hline $\mathrm{T} 2 \mathrm{HK}$ & $\geq 2.42 \times 10^{-5}$ & $\leq 2.72 \times 10^{-11}$ \\
\hline $\mathrm{T} 2 \mathrm{HKK}$ & $\geq 1.51 \times 10^{-5}$ & $\leq 4.36 \times 10^{-11}$ \\
\hline $\mathrm{ESS} \nu \mathrm{SB}$ & $\geq 2.71 \times 10^{-5}$ & $\leq 2.43 \times 10^{-11}$ \\
\hline $\mathrm{T} 2 \mathrm{HK}+\mathrm{ESS} \nu \mathrm{SB}$ & $\geq 1.51 \times 10^{-5}$ & $\leq 4.36 \times 10^{-11}$ \\
\hline $\mathrm{T} 2 \mathrm{HKK}+\mathrm{ESS} \nu \mathrm{SB}$ & $\geq 1.19 \times 10^{-5}$ & $\leq 5.53 \times 10^{-11}$ \\
\hline
\end{tabular}

Table 4. The sensitivity to the decay rate $\alpha$ and the neutrino decay lifetime at $3 \sigma$ CL for the standalone experiments T2HK, T2HKK, ESS $\nu \mathrm{SB}$ and also for the combinations of T2HK+ESS $\nu \mathrm{SB}$, $\mathrm{T} 2 \mathrm{HKK}+\mathrm{ESS} \nu \mathrm{SB}$.

In conclusion, we studied the sensitivity to decay at experiments at the first and second oscillation maxima and discussed the salient features. Overall the combination of first and second oscillation maxima is best for decay since at the first oscillation maximum the precision of $\theta_{23}$ is more and at the second oscillation maximum the sensitivity to decay could be more because of enhanced baseline (for same energy) and/or lower energy (for same baseline). Our study underscores the importance of the disappearance channel in giving enhanced octant sensitivity for second oscillation maxima experiments in presence of decay and the importance of better energy resolution in obtaining a higher sensitivity to decay.

Note added. While this work was being written a paper [72] came on arXiv, which also considered neutrino decay in the context of ESS $\nu$ SB experiment. Our study contains a comparative analysis of T2HK, T2HKK and ESS $\nu$ SB experiment. We have observed that our bounds on $\tau_{3} / m_{3}$ are slightly less constraining compared to [72], this is because we have a runtime of 2 years neutrino and 8 years antineutrino which is proposed by the $\mathrm{ESS} \nu \mathrm{SB}$ collaboration [11]. The runtime configuration was optimized for $\mathrm{CP}$ violation discovery. The authors in [72] have considered 5 years neutrino and 5 years antineutrino and having more neutrino run increases the statistics which is the reason for their results being more constraining. Our results are compatible with their results for the same runtime and same set of oscillation parameters.

\section{Acknowledgments}

D.P. is thankful for the support of FAPESP funding Grant 2014/19164-6 and 2020/042617. The authors would like to thank Enrique Fernandez-Martinez for providing them with the GLoBES files used for the simulation of the ESS $\nu \mathrm{SB}$ experiment. DD and DP would like to acknowledge the organisers of WHEPP-XVI for their hospitality where some initial discussions about the work took place.

Open Access. This article is distributed under the terms of the Creative Commons Attribution License (CC-BY 4.0), which permits any use, distribution and reproduction in any medium, provided the original author(s) and source are credited. 


\section{References}

[1] I. Esteban, M.C. Gonzalez-Garcia, M. Maltoni, T. Schwetz and A. Zhou, The fate of hints: updated global analysis of three-flavor neutrino oscillations, JHEP 09 (2020) 178 [arXiv:2007.14792] [INSPIRE].

[2] P.F. de Salas et al., 2020 global reassessment of the neutrino oscillation picture, JHEP 02 (2021) 071 [arXiv:2006.11237] [INSPIRE].

[3] F. Capozzi, E. Di Valentino, E. Lisi, A. Marrone, A. Melchiorri and A. Palazzo, Global constraints on absolute neutrino masses and their ordering, Phys. Rev. D 95 (2017) 096014 [Addendum ibid. 101 (2020) 116013] [arXiv: 2003.08511] [INSPIRE].

[4] DUNE collaboration, Long-Baseline Neutrino Facility (LBNF) and Deep Underground Neutrino Experiment (DUNE): conceptual design report, volume 2. The physics program for DUNE at LBNF, arXiv: 1512.06148 [INSPIRE].

[5] DUNE collaboration, Long-Baseline Neutrino Facility (LBNF) and Deep Underground Neutrino Experiment (DUNE): conceptual design report, volume 1. The LBNF and DUNE projects, arXiv:1601.05471 [INSPIRE].

[6] DUNE collaboration, Long-Baseline Neutrino Facility (LBNF) and Deep Underground Neutrino Experiment (DUNE): conceptual design report, volume 4. The DUNE detectors at $L B N F$, arXiv: 1601.02984 [INSPIRE].

[7] DUNE collaboration, The DUNE far detector interim design report volume 1: physics, technology and strategies, arXiv:1807.10334 [INSPIRE].

[8] DUNE collaboration, Long-Baseline Neutrino Facility (LBNF) and Deep Underground Neutrino Experiment (DUNE): conceptual design report, volume 3. Long-Baseline Neutrino Facility for DUNE June 24, 2015, arXiv:1601.05823 [INSPIRE].

[9] Hyper-Kamiokande Proto- collaboration, Physics potential of a long-baseline neutrino oscillation experiment using a J-PARC neutrino beam and Hyper-Kamiokande, PTEP 2015 (2015) $053 \mathrm{C} 02$ [arXiv:1502.05199] [INSPIRE].

[10] HyPer-Kamiokande collaboration, Physics potentials with the second Hyper-Kamiokande detector in Korea, PTEP 2018 (2018) 063C01 [arXiv:1611.06118] [INSPIRE].

[11] E. Baussan, M. Dracos, T. Ekelof, E.F. Martinez, H. Ohman and N. Vassilopoulos, The use the a high intensity neutrino beam from the ESS proton linac for measurement of neutrino CP-violation and mass hierarchy, arXiv:1212.5048 [INSPIRE].

[12] ESSNUSB collaboration, A very intense neutrino super beam experiment for leptonic CP-violation discovery based on the European spallation source linac, Nucl. Phys. B $\mathbf{8 8 5}$ (2014) 127 [arXiv:1309.7022] [INSPIRE].

[13] JUNO collaboration, Neutrino physics with JUNO, J. Phys. G 43 (2016) 030401 [arXiv: 1507.05613] [INSPIRE].

[14] ICAL collaboration, Physics potential of the ICAL detector at the India-based Neutrino Observatory (INO), Pramana 88 (2017) 79 [arXiv: 1505.07380] [INSPIRE].

[15] ICECUBE-PINGU collaboration, Letter of intent: the Precision IceCube Next Generation Upgrade (PINGU), arXiv:1401.2046 [INSPIRE].

[16] KM3NET collaboration, The KM3NeT deep-sea neutrino telescope, Nucl. Instrum. Meth. A 766 (2014) 83 [arXiv: 1408.1392] [INSPIRE]. 
[17] J.N. Bahcall, N. Cabibbo and A. Yahil, Are neutrinos stable particles?, Phys. Rev. Lett. 28 (1972) 316 [INSPIRE].

[18] A. Acker and S. Pakvasa, Solar neutrino decay, Phys. Lett. B 320 (1994) 320 [hep-ph/9310207] [INSPIRE].

[19] Z.G. Berezhiani, G. Fiorentini, M. Moretti and A. Rossi, Fast neutrino decay and solar neutrino detectors, Z. Phys. C $\mathbf{5 4}$ (1992) 581 [inSPIRE].

[20] Z.G. Berezhiani, M. Moretti and A. Rossi, Matter induced neutrino decay and solar anti-neutrinos, Z. Phys. C 58 (1993) 423 [InSPIRE].

[21] S. Choubey, S. Goswami and D. Majumdar, Status of the neutrino decay solution to the solar neutrino problem, Phys. Lett. B 484 (2000) 73 [hep-ph/0004193] [INSPIRE].

[22] A. Bandyopadhyay, S. Choubey and S. Goswami, MSW mediated neutrino decay and the solar neutrino problem, Phys. Rev. D 63 (2001) 113019 [hep-ph/0101273] [INSPIRE].

[23] A.S. Joshipura, E. Masso and S. Mohanty, Constraints on decay plus oscillation solutions of the solar neutrino problem, Phys. Rev. D 66 (2002) 113008 [hep-ph/0203181] [INSPIRE].

[24] A. Bandyopadhyay, S. Choubey and S. Goswami, Neutrino decay confronts the SNO data, Phys. Lett. B $\mathbf{5 5 5}$ (2003) 33 [hep-ph/0204173] [INSPIRE].

[25] R. Picoreti, M.M. Guzzo, P.C. de Holanda and O.L.G. Peres, Neutrino decay and solar neutrino seasonal effect, Phys. Lett. B 761 (2016) 70 [arXiv:1506.08158] [InSPIRE].

[26] J.M. Berryman, A. de Gouvêa and D. Hernandez, Solar neutrinos and the decaying neutrino hypothesis, Phys. Rev. D 92 (2015) 073003 [arXiv:1411.0308] [INSPIRE].

[27] J.A. Frieman, H.E. Haber and K. Freese, Neutrino mixing, decays and supernova SN1987A, Phys. Lett. B 200 (1988) 115 [inSPIRE].

[28] G.-Y. Huang and S. Zhou, Constraining neutrino lifetimes and magnetic moments via solar neutrinos in the large xenon detectors, JCAP 02 (2019) 024 [arXiv:1810.03877] [INSPIRE].

[29] J.M. LoSecco, What the atmospheric neutrino anomaly is not, hep-ph/9809499 [INSPIRE].

[30] V.D. Barger, J.G. Learned, S. Pakvasa and T.J. Weiler, Neutrino decay as an explanation of atmospheric neutrino observations, Phys. Rev. Lett. 82 (1999) 2640 [astro-ph/9810121] [INSPIRE].

[31] P. Lipari and M. Lusignoli, On exotic solutions of the atmospheric neutrino problem, Phys. Rev. D 60 (1999) 013003 [hep-ph/9901350] [INSPIRE].

[32] G.L. Fogli, E. Lisi, A. Marrone and G. Scioscia, Super-Kamiokande data and atmospheric neutrino decay, Phys. Rev. D 59 (1999) 117303 [hep-ph/9902267] [INSPIRE].

[33] S. Choubey and S. Goswami, Is neutrino decay really ruled out as a solution to the atmospheric neutrino problem from Super-Kamiokande data?, Astropart. Phys. 14 (2000) 67 [hep-ph/9904257] [INSPIRE].

[34] V.D. Barger, J.G. Learned, P. Lipari, M. Lusignoli, S. Pakvasa and T.J. Weiler, Neutrino decay and atmospheric neutrinos, Phys. Lett. B 462 (1999) 109 [hep-ph/9907421] [INSPIRE].

[35] SuPER-KamiokAnde collaboration, Evidence for an oscillatory signature in atmospheric neutrino oscillation, Phys. Rev. Lett. 93 (2004) 101801 [hep-ex/0404034] [INSPIRE].

[36] M.C. Gonzalez-Garcia and M. Maltoni, Status of oscillation plus decay of atmospheric and long-baseline neutrinos, Phys. Lett. B 663 (2008) 405 [arXiv:0802.3699] [INSPIRE]. 
[37] R.A. Gomes, A.L.G. Gomes and O.L.G. Peres, Constraints on neutrino decay lifetime using long-baseline charged and neutral current data, Phys. Lett. B $\mathbf{7 4 0}$ (2015) 345 [arXiv:1407.5640] [INSPIRE].

[38] S. Choubey, D. Dutta and D. Pramanik, Invisible neutrino decay in the light of NOvA and T2K data, JHEP 08 (2018) 141 [arXiv: 1805.01848] [INSPIRE].

[39] P.B. Denton and I. Tamborra, Invisible neutrino decay could resolve IceCube's track and cascade tension, Phys. Rev. Lett. 121 (2018) 121802 [arXiv:1805.05950] [INSPIRE].

[40] T. Abrahão, H. Minakata, H. Nunokawa and A.A. Quiroga, Constraint on neutrino decay with medium-baseline reactor neutrino oscillation experiments, JHEP 11 (2015) 001 [arXiv: 1506.02314] [INSPIRE].

[41] J.F. Beacom, N.F. Bell, D. Hooper, S. Pakvasa and T.J. Weiler, Decay of high-energy astrophysical neutrinos, Phys. Rev. Lett. 90 (2003) 181301 [hep-ph/0211305] [INSPIRE].

[42] M. Maltoni and W. Winter, Testing neutrino oscillations plus decay with neutrino telescopes, JHEP 07 (2008) 064 [arXiv:0803.2050] [INSPIRE].

[43] S. Pakvasa, A. Joshipura and S. Mohanty, Explanation for the low flux of high energy astrophysical muon-neutrinos, Phys. Rev. Lett. 110 (2013) 171802 [arXiv:1209.5630] [INSPIRE].

[44] G. Pagliaroli, A. Palladino, F.L. Villante and F. Vissani, Testing nonradiative neutrino decay scenarios with IceCube data, Phys. Rev. D 92 (2015) 113008 [arXiv:1506.02624] [INSPIRE].

[45] M. Bustamante, J.F. Beacom and K. Murase, Testing decay of astrophysical neutrinos with incomplete information, Phys. Rev. D 95 (2017) 063013 [arXiv: 1610.02096] [INSPIRE].

[46] S. Choubey, S. Goswami and D. Pramanik, A study of invisible neutrino decay at DUNE and its effects on $\theta_{23}$ measurement, JHEP 02 (2018) 055 [arXiv: 1705.05820] [INSPIRE].

[47] A. Ghoshal, A. Giarnetti and D. Meloni, Neutrino invisible decay at DUNE: a multi-channel analysis, J. Phys. G 48 (2021) 055004 [arXiv: 2003.09012] [inSPIRE].

[48] J. Tang, T.-C. Wang and Y. Zhang, Invisible neutrino decays at the MOMENT experiment, JHEP 04 (2019) 004 [arXiv: 1811.05623] [INSPIRE].

[49] S. Choubey, S. Goswami, C. Gupta, S.M. Lakshmi and T. Thakore, Sensitivity to neutrino decay with atmospheric neutrinos at the INO-ICAL detector, Phys. Rev. D 97 (2018) 033005 [arXiv: 1709.10376] [INSPIRE].

[50] L.S. Mohan, Probing the sensitivity to leptonic $\delta_{C P}$ in presence of invisible decay of $\nu_{3}$ using atmospheric neutrinos, J. Phys. G 47 (2020) 115004 [arXiv:2006.04233] [INSPIRE].

[51] P.F. de Salas, S. Pastor, C.A. Ternes, T. Thakore and M. Tórtola, Constraining the invisible neutrino decay with KM3NeT-ORCA, Phys. Lett. B 789 (2019) 472 [arXiv:1810.10916] [INSPIRE].

[52] A. Acker, S. Pakvasa and J.T. Pantaleone, Decaying Dirac neutrinos, Phys. Rev. D 45 (1992) 1 [INSPIRE].

[53] G.B. Gelmini and M. Roncadelli, Left-handed neutrino mass scale and spontaneously broken lepton number, Phys. Lett. B 99 (1981) 411 [INSPIRE].

[54] Y. Chikashige, R.N. Mohapatra and R.D. Peccei, Are there real Goldstone bosons associated with broken lepton number?, Phys. Lett. B 98 (1981) 265 [INSPIRE]. 
[55] S. Pakvasa, Do neutrinos decay?, AIP Conf. Proc. 542 (2000) 99 [hep-ph/0004077] [INSPIRE].

[56] C.W. Kim and W.P. Lam, Some remarks on neutrino decay via a Nambu-Goldstone boson, Mod. Phys. Lett. A 5 (1990) 297 [INSPIRE].

[57] A. Acker, A. Joshipura and S. Pakvasa, A neutrino decay model, solar anti-neutrinos and atmospheric neutrinos, Phys. Lett. B 285 (1992) 371 [INSPIRE].

[58] M. Lindner, T. Ohlsson and W. Winter, A combined treatment of neutrino decay and neutrino oscillations, Nucl. Phys. B 607 (2001) 326 [hep-ph/0103170] [INSPIRE].

[59] P. Coloma and O.L.G. Peres, Visible neutrino decay at DUNE, arXiv:1705.03599 [INSPIRE].

[60] A.M. Gago, R.A. Gomes, A.L.G. Gomes, J. Jones-Perez and O.L.G. Peres, Visible neutrino decay in the light of appearance and disappearance long baseline experiments, JHEP 11 (2017) 022 [arXiv : 1705.03074] [inSPIRE].

[61] M.V. Ascencio-Sosa, A.M. Calatayud-Cadenillas, A.M. Gago and J. Jones-Pérez, Matter effects in neutrino visible decay at future long-baseline experiments, Eur. Phys. J. C 78 (2018) 809 [arXiv : 1805. 03279] [INSPIRE].

[62] Y.P. Porto-Silva, S. Prakash, O.L.G. Peres, H. Nunokawa and H. Minakata, Constraining visible neutrino decay at KamLAND and JUNO, Eur. Phys. J. C 80 (2020) 999 [arXiv: 2002.12134] [INSPIRE].

[63] A. Abdullahi and P.B. Denton, Visible decay of astrophysical neutrinos at IceCube, Phys. Rev. D 102 (2020) 023018 [arXiv:2005.07200] [inSPIRE].

[64] M. Escudero and M. Fairbairn, Cosmological constraints on invisible neutrino decays revisited, Phys. Rev. D 100 (2019) 103531 [arXiv: 1907.05425] [INSPIRE].

[65] Super-Kamiokande collaboration, The Super-Kamiokande detector, Nucl. Instrum. Meth. A $\mathbf{5 0 1}$ (2003) 418 [INSPIRE].

[66] T. Kajita, H. Minakata, S. Nakayama and H. Nunokawa, Resolving eight-fold neutrino parameter degeneracy by two identical detectors with different baselines, Phys. Rev. D 75 (2007) 013006 [hep-ph/0609286] [INSPIRE].

[67] P. Huber, M. Lindner and W. Winter, Simulation of long-baseline neutrino oscillation experiments with GLoBES (General Long Baseline Experiment Simulator), Comput. Phys. Commun. 167 (2005) 195 [hep-ph/0407333] [INSPIRE].

[68] P. Huber, J. Kopp, M. Lindner, M. Rolinec and W. Winter, New features in the simulation of neutrino oscillation experiments with GLoBES 3.0: General Long Baseline Experiment Simulator, Comput. Phys. Commun. 177 (2007) 432 [hep-ph/0701187] [INSPIRE].

[69] MEMPHYS collaboration, Study of the performance of a large scale water-Cherenkov detector (MEMPHYS), JCAP 01 (2013) 024 [arXiv: 1206.6665] [INSPIRE].

[70] NuFIT version 5.0 webpage, , (2020).

[71] A. Bandyopadhyay, S. Choubey and S. Goswami, Exploring the sensitivity of current and future experiments to $\theta_{\text {solar }}$, Phys. Rev. D 67 (2003) 113011 [hep-ph/0302243] [INSPIRE].

[72] S. Choubey, M. Ghosh, D. Kempe and T. Ohlsson, Exploring invisible neutrino decay at $E S S \nu S B$, arXiv:2010.16334 [INSPIRE]. 